\title{
Rational solutions to the Pfaff lattice and Jack polynomials
}

\author{
M. Adler* V. B. Kuznetsov ${ }^{\dagger} \quad$ P. van Moerbeke ${ }^{\ddagger}$ \\ February 15, 2002
}

To the memory of Jürgen Moser

\begin{abstract}
The finite Pfaff lattice is given by commuting Lax pairs involving a finite matrix $L$ (zero above the first subdiagonal) and a projection onto $S p(N)$. The lattice admits solutions such that the entries of the matrix $L$ are rational in the time parameters $t_{1}, t_{2}, \ldots$, after conjugation by a diagonal matrix. The sequence of polynomial $\tau$-functions, solving the problem, belongs to an intriguing chain of subspaces of Schur polynomials, associated to Young diagrams, dual with respect to a finite chain of rectangles. Also, this sequence of $\tau$-functions is given inductively by the action of a fixed vertex operator.

As examples, one such sequence is given by Jack polynomials for rectangular Young diagrams, while another chain starts with any twocolumn Jack polynomial.
\end{abstract}

*Department of Mathematics, Brandeis University, Waltham, Mass 02454, USA. Email: adler@brandeis.edu. The support of a National Science Foundation grant \# DMS01-00782 is gratefully acknowledged.

${ }^{\dagger}$ Department of Applied Mathematics, University of Leeds, Leeds LS2 9JT. E-mail: V.B.Kuznetsov@leeds.ac.uk. The support of a FSR grant, Universite de Louvain, Belgium, and from the EPSRC, UK, is gratefully acknowledged. This work was done, while visiting the University of Louvain and Brandeis University.

‡Department of Mathematics, Université de Louvain, 1348 Louvain-la-Neuve, Belgium and Brandeis University, Waltham, Mass 02454, USA. E-mail: vanmoerbeke@geom.ucl.ac.be and @brandeis.edu. The support of a National Science Foundation grant \# DMS-01-00782, a Nato, a FNRS and a Francqui Foundation grant is gratefully acknowledged. 


\section{Contents}

\begin{tabular}{lll}
\hline 1 & Introduction & 2
\end{tabular}

2 The vector fields $\partial m / \partial t_{k}=\Lambda^{k} m+m \Lambda^{\top k}$ and the finite Pfaff $\begin{array}{ll}\text { lattice } & 12\end{array}$

3 The solution to the Pfaff lattice with anti-diagonal skewsymmetric initial condition

$\begin{array}{|ll|}4 & \text { Proof of Theorem 1.1 }\end{array}$

\begin{tabular}{|ll}
5 & Vertex operators \\
\hline
\end{tabular}

$\begin{array}{lll}6 & \text { Duality } & 43\end{array}$

\begin{tabular}{lll}
\hline 7 & Examples & 46
\end{tabular}

\section{Introduction}

Self-dual partitions: For positive integer $n$ and $n \mid k$, define the following sets of partitions,

$$
\begin{aligned}
\mathbb{Y} & =\left\{\lambda=\left(\lambda_{1}, \lambda_{2}, \ldots\right), \quad \lambda_{1} \geq \lambda_{2} \geq \cdots \geq 0\right\} \\
\mathbb{Y}_{k} & =\left\{\lambda \in \mathbb{Y},|\lambda|=\sum \lambda_{i}=k\right\} \\
\mathbb{Y}_{k}^{(n)} & =\left\{\begin{array}{cc}
\lambda=\left(\lambda_{1}, \lambda_{2}, \ldots, \lambda_{n}\right) \in \mathbb{Y}_{k}, & \hat{\lambda}_{1} \leq n, \\
\lambda_{i}+\lambda_{n+1-i}=\frac{2 k}{n}, & 1 \leq i \leq\left[\frac{n+1}{2}\right]
\end{array}\right\},
\end{aligned}
$$

with

$$
\# \mathbb{Y}_{k}^{(n)}=\left(\begin{array}{c}
{\left[\frac{n}{2}+\frac{k}{n}\right]} \\
{\left[\frac{n+1}{2}\right]}
\end{array}\right)
$$

These are a few examples:
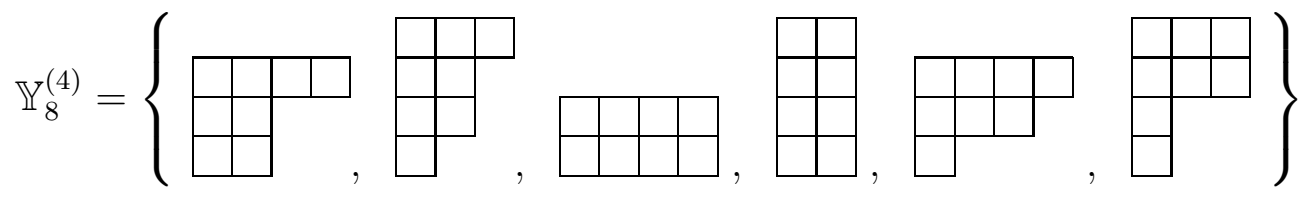


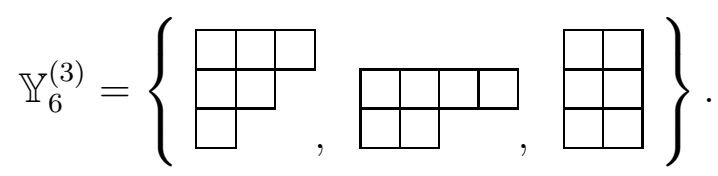

Let $\mathbf{s}_{\lambda}(t):=\operatorname{det}\left(s_{\lambda_{i}-i+j}(t)\right)_{1 \leq i, j}$ be the Schur polynomials corresponding to $\lambda$, with $\mathbf{s}_{i}(t)$ being the elementary Schur polynomials, defined by

$$
e^{\sum_{1}^{\infty} t_{i} z^{i}}=\sum_{i \geq 0} \mathbf{s}_{i}(t) z^{i} \quad \text { with } \quad \mathbf{s}_{i}(t)=0 \text { for } i<0 .
$$

The linear space

$$
\mathbb{L}_{k}^{(n)}:=\left\{\sum_{\lambda \in \mathbb{Y}_{k}^{(n)}} a_{\lambda} \mathbf{s}_{\lambda} \mid a_{\lambda} \in \mathbb{C}\right\}
$$

will play an ubiquitous role in this work.

The finite Pfaff Lattice: The $N \times N$ skew-symmetric matrices,

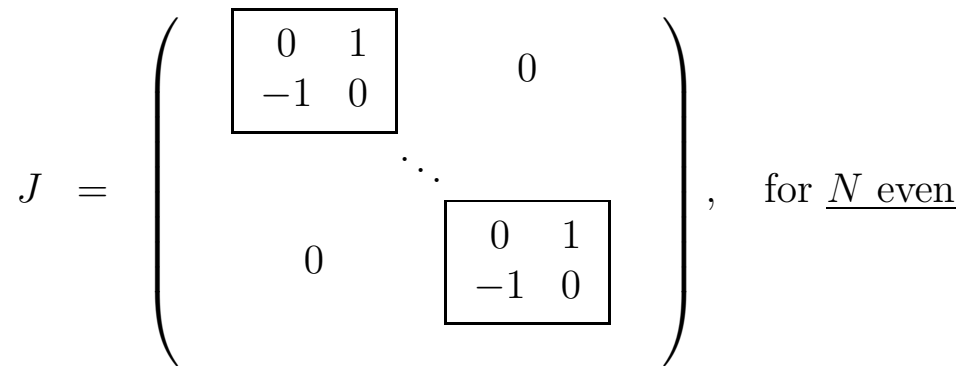

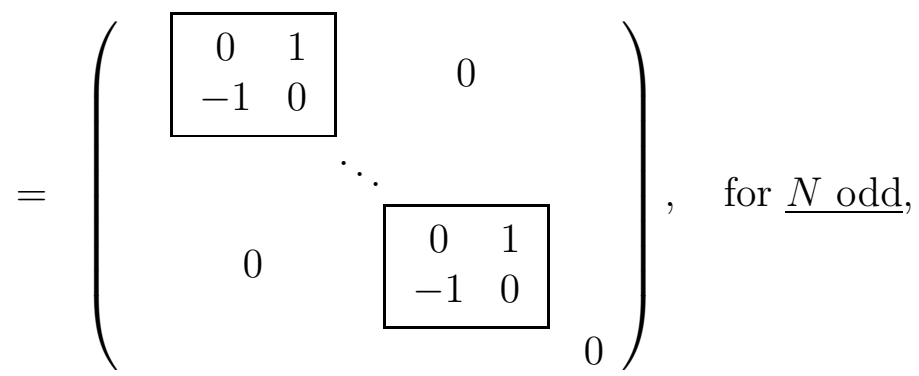

satisfy

$$
J^{2}=\left\{\begin{array}{l}
-I_{N}, \text { for } N \text { even } \\
\left(\begin{array}{cc}
-I_{N-1} & O \\
O & 0
\end{array}\right), \text { for } N \text { odd }
\end{array}\right.
$$


Also consider the Lie algebra $\mathfrak{k}$ of lower-triangular matrices of the form

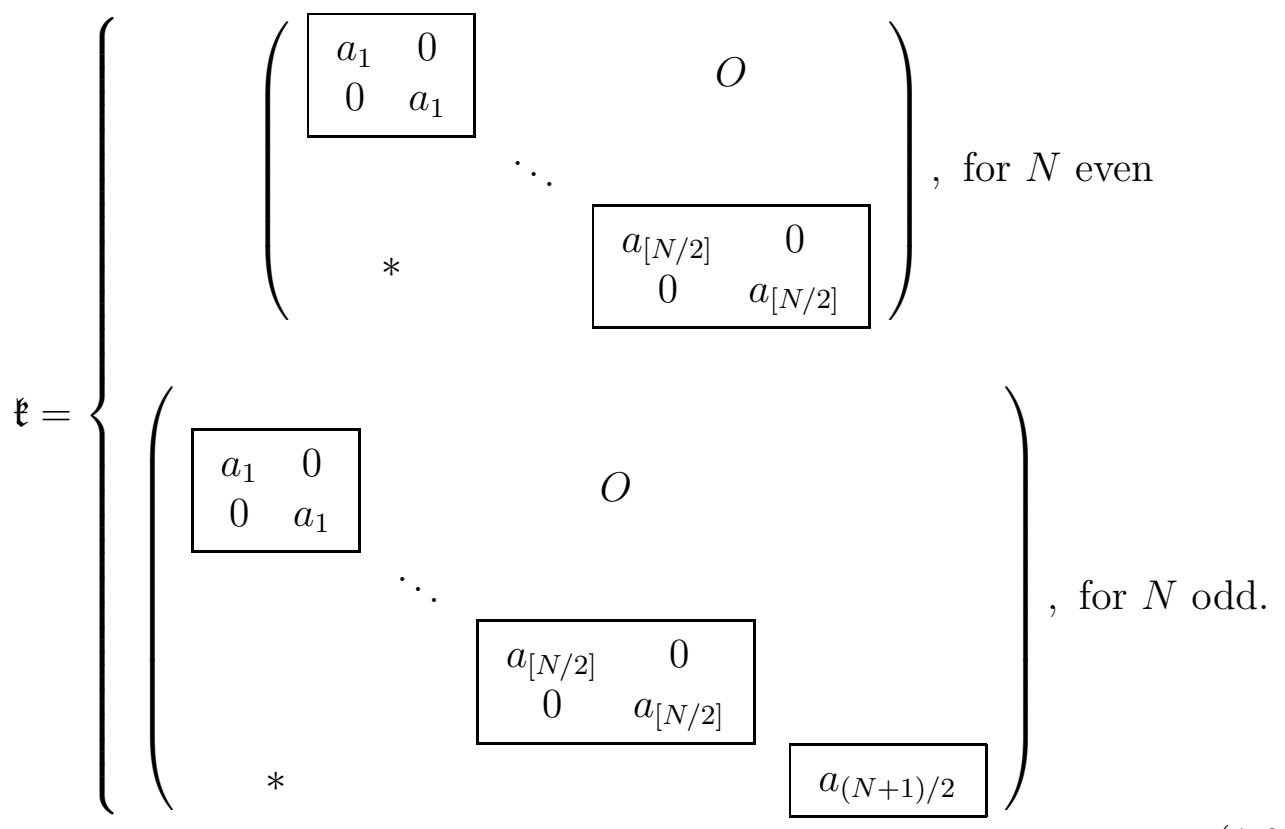

For each $a \in g l(N)$, consider the decomposition!

$$
\begin{aligned}
a= & (a)_{\mathfrak{k}}+(a)_{\mathfrak{n}} \\
= & \pi_{\mathfrak{k}} a+\pi_{\mathfrak{n}} a \\
= & \left(\left(a_{-}-J\left(a_{+}\right)^{\top} J\right)+\frac{1}{2}\left(a_{0}-J\left(a_{0}\right)^{\top} J\right)\right) \\
& \quad+\left(\left(a_{+}+J\left(a_{+}\right)^{\top} J\right)+\frac{1}{2}\left(a_{0}+J\left(a_{0}\right)^{\top} J\right)\right) .
\end{aligned}
$$

For $N$ even, this corresponds to a Lie algebra splitting, given by

$$
g l(N)=\mathfrak{k}+\mathfrak{n}\left\{\begin{array}{l}
\mathfrak{k}=\{\text { lower-triangular matrices of the form }(1.0 .3)\} \\
\mathfrak{n}=s p(N)=\left\{a \text { such that } J a^{\top} J=a\right\}
\end{array}\right.
$$

\footnotetext{
${ }^{1} a_{ \pm}$refers to projection onto strictly upper (strictly lower) triangular matrices, with all $2 \times 2$ diagonal blocks equal zero. $a_{0}$ refers to projection onto the "diagonal", consisting of $2 \times 2$ blocks.
} 
For $N$ odd, this is merely a vector space splitting $g l(N)=\mathfrak{k}+\mathfrak{n}\left\{\begin{array}{l}\mathfrak{k}=\{\text { lower-triangular matrices of the form (1.0.3) }\} \\ \mathfrak{n}=\operatorname{span}\left\{\pi_{\mathfrak{n}}(a) \text { with } a \in \operatorname{gl}(N)\right\} .\end{array}\right.$

The Pfaff lattice is defined on $N \times N$ matrices $L$ of the form

$$
\begin{aligned}
L= & \left(\begin{array}{cccccccc}
0 & 1 & & & & & & \\
& -d_{1} & a_{1} & & & & & \\
& & d_{1} & 1 & & & & \\
& & & -d_{2} & a_{2} & & & \\
& & & & d_{2} & & & \\
& * & & & & \ddots & a_{\frac{N-2}{2}} & \\
& & & & & & -d_{\frac{N-2}{2}} & 1 \\
0 & & & & & & & 0
\end{array}\right), \text { for } N \text { even } \\
& =\left(\begin{array}{cccccccc}
0 & 1 & & & & & & \\
& -d_{1} & a_{1} & & & & & \\
& & d_{1} & 1 & & & & \\
& & & -d_{2} & a_{2} & & & \\
& * & & & d_{2} & & & \\
& & & & & & -d_{\frac{N-1}{2}} & a_{\frac{N-1}{2}} \\
& & & & & & & d_{\frac{N-1}{2}}
\end{array}\right), \text { for } N \text { odd. }
\end{aligned}
$$

namely,

$$
\frac{\partial L}{\partial t_{i}}=\left[-\left(L^{i}\right)_{\mathfrak{k}}, L\right] . \quad \text { (Pfaff lattice) }
$$

Given arbitrary, but fixed parameters

$$
b_{0}, \ldots, b_{\left[\frac{N-2}{2}\right]} \in \mathbb{C}
$$


consider the skew-symmetric antidiagonal initial condition,

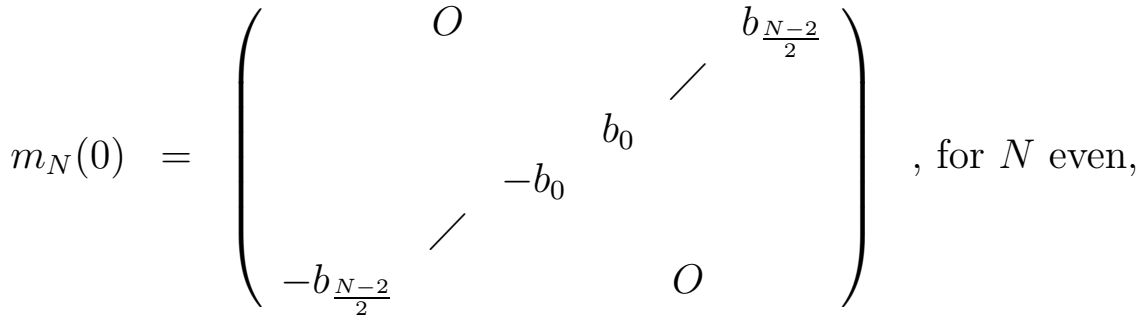

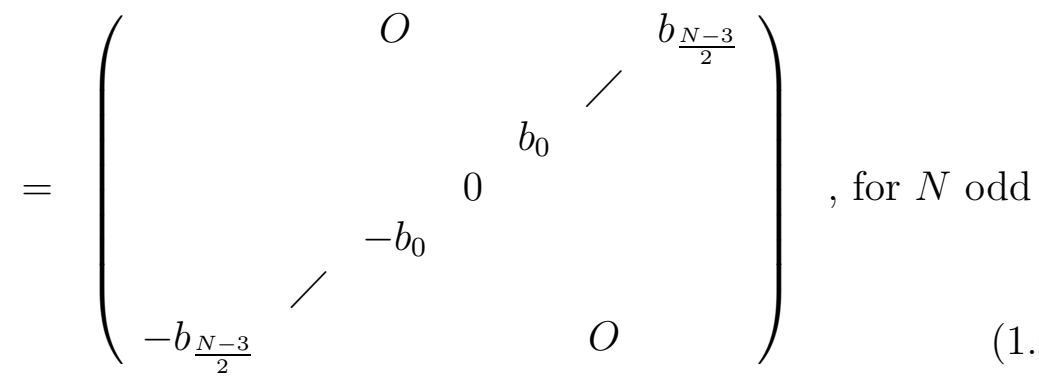

and its time evolution (respecting the skew-symmetry),

$$
m_{\ell}(t)=E_{\ell, N}(t) m_{N}(0) E_{\ell, N}^{\top}(t),
$$

where?

$$
E_{\ell, N}(t):=\left(e^{\sum_{1}^{\infty} t_{i} \Lambda^{i}}\right)_{\substack{1, \ldots, \ell \\ 1, \ldots, N}}
$$

The Pfaffian $p f m_{\ell}(t)$ of the skew-symmetric matrix $m_{\ell}(t)$ will play an important role in this paper.

\section{Rational solutions to the Pfaff Lattice:}

Theorem 1.1 Modulo conjugation by a $N \times N$ diagonal matrix $D(t)$ (see remark below), the finite Pfaff lattice

$$
\frac{\partial L}{\partial t_{i}}=\left[-\left(L^{i}\right)_{\mathfrak{k}}, L\right] . \quad \text { (Pfaff lattice) }
$$

has rational solutions in $t_{1}, t_{2}, \ldots$; i.e., the matrix

$$
D^{-1}(t) L(t) D(t)=\tilde{Q}(t) \Lambda \tilde{Q}(t)^{-1}
$$

\footnotetext{
${ }^{2} \Lambda$ is the finite shift matrix $\Lambda:=\left(\delta_{i, j-1}\right)_{1 \leq i, j \leq N}$ and $(A)_{\substack{1, \ldots, \ell \\ 1, \ldots, N}}$ denotes the matrix formed by the first $\ell$ rows and first $N$ columns of $A$.
} 
is rational in $t_{1}, t_{2}, \ldots$, with $\tilde{Q}(t)$ a lower-triangular $N \times N$ matrix with rational entries, obtained by Taylor expanding $\tau_{2 n}\left(t-\left[z^{-1}\right]\right)$ in $z^{-1}$, with $\tau_{0}=1$,

$$
\begin{aligned}
\tilde{q}_{2 n}(t ; z) & :=\sum_{j=0}^{2 n} \tilde{Q}_{2 n+1, j+1}(t) z^{j}=z^{2 n} \tau_{2 n}\left(t-\left[z^{-1}\right]\right) \quad \text { with } 0 \leq n \leq\left[\frac{N-1}{2}\right] \\
\tilde{q}_{2 n+1}(t ; z) & :=\sum_{j=0}^{2 n+1} \tilde{Q}_{2 n+2, j+1}(t) z^{j}=z^{2 n}\left(z+\frac{\partial}{\partial t_{1}}\right) \tau_{2 n}\left(t-\left[z^{-1}\right]\right),
\end{aligned}
$$

with (see the definition of the $\mathbb{L}$-space in the beginning of this section)

$$
\begin{aligned}
\tau_{\ell}(t) & =p f\left(E_{\ell, N}(t) m_{N}(0) E_{\ell, N}^{\top}(t)\right) \\
& =\sum_{\lambda \in \mathbb{Y}_{\frac{\ell(N-\ell)}{(\ell)}}}\left(\prod_{1}^{[\ell / 2]} b_{\lambda_{i}-i+\ell-\left[\frac{N+1}{2}\right]}\right) \mathbf{s}_{\lambda}(t), \quad \text { for }\left\{\begin{array}{l}
0 \leq \ell \leq N-1 \\
\ell \text { even }
\end{array}\right. \\
& \in \mathbb{L}_{\frac{\ell(N-\ell)}{2}}^{(\ell)} .
\end{aligned}
$$

The polynomials $q_{k}=D_{k} \tilde{q}_{k}$ (in z) of degree $0 \leq k \leq N-1$ are "skeworthonormal" with respect to the skew inner-product $\left\langle z^{i}, z^{j}\right\rangle=m_{i j}(t)$, i.e.,

$$
\left\langle q_{i}, q_{j}\right\rangle=J_{i j}
$$

and the $N$-vector $\left(q_{0}, \ldots, q_{N-1}\right)^{\top}$ is an eigenvector for the matrix $L$, with modified boundary conditions. The fact that $Q_{2 n, 2 n-1}=0$ defines the skeworthogonal polynomials in a unique way, up to \pm 1 .

Example: For $\ell=2$, we have

$$
\begin{aligned}
\tau_{2}(t) & =\sum_{i=0}^{\frac{N-2}{2}} b_{i} \mathbf{s}_{\frac{N-2}{2}+i, \frac{N-2}{2}-i}(t), \quad \text { for } N \text { even }, \\
& =\sum_{i=0}^{\frac{N-3}{2}} b_{i} \mathbf{s}_{\frac{N-1}{2}+i, \frac{N-3}{2}-i}(t), \quad \text { for } N \text { odd } .
\end{aligned}
$$




\section{Remark:}

$D(t)$

$$
\begin{aligned}
& =\operatorname{diag}\left(\frac{1}{\sqrt{\tau_{0} \tau_{2}}}, \frac{1}{\sqrt{\tau_{0} \tau_{2}}}, \frac{1}{\sqrt{\tau_{2} \tau_{4}}}, \frac{1}{\sqrt{\tau_{2} \tau_{4}}}, \ldots, \frac{1}{\sqrt{\tau_{N-2} \tau_{N}}}, \frac{1}{\sqrt{\tau_{N-2} \tau_{N}}}\right) \text { for } \mathrm{N} \text { even } \\
& =\operatorname{diag}\left(\frac{1}{\sqrt{\tau_{0} \tau_{2}}}, \frac{1}{\sqrt{\tau_{0} \tau_{2}}}, \ldots, \frac{1}{\sqrt{\tau_{N-3} \tau_{N-1}}}, \frac{1}{\sqrt{\tau_{N-3} \tau_{N-1}}}, \frac{1}{\sqrt{\tau_{N-1}}}\right) \quad \text { for } \mathrm{N} \text { odd }
\end{aligned}
$$

Duality: For the case of odd $N$, we can even define $\tau_{\ell}(t)$ for odd $\ell$, by slightly deforming the initial moment matrix $m_{N}(0)$. In section 6 , we prove a duality between these $\tau_{k}$ 's for $k$ even and odd, as follows

$$
\tilde{\tau}_{\ell}(t)=(-1)^{\ell(N-\ell) / 2}\left(\prod_{0}^{\frac{N-3}{2}} b_{i}\right)\left(\left.\tau_{N-\ell}(-t)\right|_{b_{i} \rightarrow b_{i}^{-1}}\right), \quad \text { for } \ell \text { odd } .
$$

\section{Fay identities:}

Theorem 1.2 The sequence of functions

$$
\tau_{\ell}(t)=\sum_{\lambda \in \mathbb{Y}_{\frac{\ell(N-\ell)}{2}}^{(\ell)}}\left(\prod_{1}^{[\ell / 2]} b_{\lambda_{i}-i+\ell-\left[\frac{N+1}{2}\right]}\right) \mathbf{s}_{\lambda}(t), \quad \begin{aligned}
& \ell \leq \ell \leq N-1 \\
& \ell \text { even }
\end{aligned}
$$

together with the "boundary condition"

$$
\tau_{0}=1 \text { and } \begin{cases}\tau_{N}=\prod_{0}^{\frac{N-2}{2}} b_{i}, & \text { for even } N \\ \tau_{N+1}=0, & \text { for odd } N\end{cases}
$$

satisfies the the "differential Fay identity":

$$
\begin{aligned}
& \left\{\tau_{2 n}(t-[u]), \tau_{2 n}(t-[v])\right\} \\
& \quad+\left(u^{-1}-v^{-1}\right)\left(\tau_{2 n}(t-[u]) \tau_{2 n}(t-[v])-\tau_{2 n}(t) \tau_{2 n}(t-[u]-[v])\right) \\
& \quad=u v(u-v) \tau_{2 n-2}(t-[u]-[v]) \tau_{2 n+2}(t) .
\end{aligned}
$$

\footnotetext{
${ }^{3}$ Define the Wronskian $\{f, g\}=\frac{\partial f}{\partial t_{1}} g-\frac{\partial g}{\partial t_{1}} f$.
} 
Vertex operator constructions of the rational solutions: Consider the vertex operator acting on functions $f(t)$ of $t=\left(t_{1}, t_{2}, \ldots\right) \in \mathbb{C}^{\infty}$, namely

$$
X(t ; z)=e^{\sum_{1}^{\infty} t_{i} z^{i}} e^{-\sum_{1}^{\infty} \frac{z^{-i}}{i} \frac{\partial}{\partial t_{i}}}
$$

and the vector vertex operator

$$
\mathbb{X}(t ; z)=\Lambda^{\top} e^{\sum_{1}^{\infty} t_{i} z^{i}} e^{-\sum_{1}^{\infty} \frac{z^{-i}}{i} \frac{\partial}{\partial t_{i}}} \chi(z),
$$

acting on vectors of functions $F=\left(f_{0}(t), f_{1}(t), \ldots\right)$, with $\chi(z):=\left(z^{i}\right)_{i \geq 0}$. Then the composition $\mathbb{X}(t ; \lambda) \mathbb{X}(t ; \mu)$ is a vertex operator for the Pfaff lattice, i.e., for any $\tau$-vector $=\left(\tau_{0}, \tau_{2}, \tau_{4}, \ldots\right)$ of the Pfaff lattice,

$$
\tau(t)+a \mathbb{X}(t ; \mu) \mathbb{X}(t ; \lambda) \tau(t), \quad a \in \mathbb{C}
$$

is again a $\tau$-vector of the Pfaff lattice, or coordinatewise

$$
\tau_{2 n}+a\left(1-\frac{\lambda}{\mu}\right) \mu^{2 n-1} \lambda^{2 n-2} e^{\sum t_{i}\left(\lambda^{i}+\mu^{i}\right)} \tau_{2 n-2}\left(t-\left[\lambda^{-1}\right]-\left[\mu^{-1}\right]\right)
$$

provides a new sequence of Pfaff $\tau$-functions.

In terms of the distributional weight, with the $b_{i}$ as in (1.0.9),

$$
\rho_{b}(x):=\left\{\begin{array}{l}
\rho_{b}^{(e)}(x)=\sum_{i \geq 0} b_{i}\left(x^{-i-1}-x^{i}\right), \text { for } N \text { even } \\
\rho_{b}^{(0)}(x)=x^{-1 / 2} \sum_{i \geq 0} b_{i}\left(x^{-i-1}-x^{i+1}\right), \text { for } N \text { odd }
\end{array}\right.
$$

and

$$
\beta:=\frac{N}{2}-\ell+1
$$

we define the integrated vertex operator, in terms of the vertex operator (1.0.21),

$$
Y_{\beta}(t):=\frac{1}{(2 \pi i)^{2}} \oint_{\infty} \oint_{\infty} X(t ; y) X(t ; z) \frac{\rho_{b}(y / z) d y d z}{z^{2}(y z)^{\beta}}
$$


and the integrated vector vertex operator, in terms of the (1.0.22),

$$
\mathbb{Y}_{N}(t)=\frac{1}{(2 \pi i)^{2}} \oint_{\infty} \oint_{\infty} \mathbb{X}(t ; y) \mathbb{X}(t ; z) \frac{\rho_{b}(y / z) d y d z}{2(y z)^{N / 2} z}
$$

In both cases, the double integral around two contours about $\infty$ amounts to computing the coefficient of $1 / y z$.

Theorem 1.3 For a given set of $b_{i}$, the sequence of $\tau$-functions $\tau_{0}, \tau_{2}, \tau_{4}, \ldots$, defined in (1.0.15), is generated by the vertex operators $Y_{p}$; to be precise, inductively

$$
Y_{\frac{N}{2}-\ell+1} \tau_{\ell-2}=\ell \tau_{\ell}
$$

Corollary 1.4 The vector of $\tau$-functions

$$
I=\left(I_{0}, I_{2}, I_{4}, \ldots\right), \text { with } I_{\ell}=\left(\frac{\ell}{2}\right) ! \tau_{\ell}
$$

is a fixed point for the vertex operator $\mathbb{Y}_{N}$, namely

$$
\left(\mathbb{Y}_{N} I\right)_{\ell}=I_{\ell}, \text { for even } \ell \text {. }
$$

The rational solutions to the Pfaff lattice can be $q$-deformed; this will be reported on at a later stage.

\section{Example 1: Rectangular Jack polynomials}

Jack polynomials are symmetric polynomials in the variables $x_{i}$, which are orthogonal with respect to the inner-product

$$
\left\langle p_{\lambda}, p_{\mu}\right\rangle=\delta_{\lambda \mu}\left(1^{m_{1}} 2^{m_{2}} \ldots\right) m_{1} ! m_{2} ! \ldots \alpha^{\lambda_{1}^{\top}}
$$

where $m_{i}=m_{i}(\lambda)$ is the number of times that $i$ appears in the partition $\lambda$ and where

$$
p_{\lambda}\left(x_{1}, x_{2}, \ldots\right):=p_{\lambda_{1}} p_{\lambda_{2}} \cdots=\sum_{i} x_{i}^{\lambda_{1}} \sum_{i} x_{i}^{\lambda_{2}} \cdots
$$

Precise definitions and properties of Jack polynomials can be found in [8, 9, 4, 6, 7]. 
Proposition 1.5 When

$$
\begin{aligned}
b_{i} & =2 i+1 \text { for } N \text { even } \\
& =2 i+2 \text { for } N \text { odd }
\end{aligned}
$$

then the $\tau_{2 n}(t)$ 's are Jack polynomials for rectangular partitions

$$
\begin{aligned}
\tau_{2 n}(t) & =\sum_{\lambda \in \mathbb{Y}_{n(N-2 n)}^{(2 n)}} \prod_{1}^{n}\left(k_{i}-k_{2 n+1-i}\right) \mathbf{s}_{\lambda}(t), \text { where }\left\{\begin{array}{l}
k_{i}=\lambda_{i}-i+2 n \\
0 \leq 2 n \leq N,
\end{array}\right. \\
& =p f m_{2 n}(t) \\
& =\frac{1}{n !} \int_{\mathbb{R}^{n}} \Delta(z)^{4} \prod_{k=1}^{n} e^{2 \sum_{1}^{\infty} t_{i} z_{k}^{i}} \delta_{\left(z_{k}\right)}^{(N-2)} d z_{k} \\
& =\left.J_{\lambda}^{(1 / 2)}(x)\right|_{t_{i}=\frac{1}{i} \sum_{k} x_{k}^{i}} \text { for } \lambda=\underbrace{(N-2 n, \ldots, N-2 n)}_{n}
\end{aligned}
$$

where the $m_{2 n}(t)$ 's are the $2 n \times 2 n$ upper-left hand corners of

$$
m_{N}(t)=\left((j-i) \tilde{\mathbf{s}}_{N-i-j-1}\right)_{0 \leq i, j \leq N-1} .
$$

upon setting $\tilde{\mathbf{s}}_{n}(t):=\mathbf{s}_{n}(2 t)$.

\section{Example 2: Two-row Jack polynomials}

Proposition 1.6 For even N, choosing

$$
\begin{gathered}
\left\{\begin{array}{l}
b_{0}=\ldots=b \frac{p}{2}-1=0 \\
b_{\frac{p}{2}+k}=\frac{(1-\alpha)_{k}(p+1)_{k}}{k !(\alpha+p+1)_{k}}, \text { for } k=0, \ldots, \frac{N-2-p}{2},
\end{array}\right. \\
{ }^{4}(a)_{k}=\frac{\Gamma(a+k)}{\Gamma(a)}=a(a+1) \ldots(a+k-1)
\end{gathered}
$$


one finds the most general two-row Jack polynomial for $\tau_{2}$, for arbitrary $\alpha$,

$$
\begin{aligned}
\tau_{2}(t) & =p f m_{2}(t) \\
& =J_{\left(\frac{N+p-2}{2}, \frac{N-p-2}{2}\right)}^{(1 / \alpha)}(t / \alpha) \\
& =c \oint \frac{d x}{2 \pi i} \frac{d y}{2 \pi i} \frac{(y-x)^{2 \alpha}}{(x y)^{\alpha+\frac{N}{2}}} e^{\sum_{1}^{\infty} t_{i}\left(x^{i}+y^{i}\right)}\left(\frac{x}{y}\right)^{p / 2}{ }_{2} F_{1}\left(\alpha,-p ; 1-\alpha-p ; \frac{y}{x}\right) .
\end{aligned}
$$

Then $\tau_{\ell}(t)$ for $\ell \geq 4$ is given by an integral of the same hypergeometric function in the integrand above.

Acknowledgment: We thank Michael Kleber for useful discussions and insights.

\section{The vector fields $\partial m / \partial t_{k}=\Lambda^{k} m+m \Lambda^{\top k}$ and the finite Pfaff lattice}

The $\ell \times N$ matrix defined in (1.0.12) reads

$$
E_{\ell, N}(t)=\left(\begin{array}{ccccc|ccc}
1 & \mathbf{s}_{1}(t) & \mathbf{s}_{2}(t) & \ldots & \mathbf{s}_{\ell-1}(t) & \mathbf{s}_{\ell}(t) & \ldots & \mathbf{s}_{N-1}(t) \\
0 & 1 & \mathbf{s}_{1}(t) & \ldots & \mathbf{s}_{\ell-2}(t) & \mathbf{s}_{\ell-1}(t) & \ldots & \mathbf{s}_{N-2}(t) \\
\vdots & \vdots & & & \vdots & \vdots & & \vdots \\
0 & 0 & 0 & \ldots & \mathbf{s}_{1}(t) & \mathbf{s}_{2}(t) & \ldots & \mathbf{s}_{N-\ell+1}(t) \\
0 & 0 & 0 & \ldots & 1 & \mathbf{s}_{1}(t) & \ldots & \mathbf{s}_{N-\ell}(t)
\end{array}\right)
$$

The main claim of this section can be summarized in the following statement:

Proposition 2.1 The commuting equations (for definition of $\Lambda$, see footnote 2)

$$
\frac{\partial m_{N}}{\partial t_{k}}=\Lambda^{k} m_{N}+m_{N} \Lambda^{\top k}
$$


with $N \times N$ skew-symmetric initial condition $m(0)$, have the following solution

$$
m_{N}(t)=E_{N, N}(t) m_{N}(0) E_{N, N}^{\top}(t) .
$$

In particular, each $\ell \times \ell$ upper-left block of $m(t)$ equals

$$
m_{\ell}(t)=E_{\ell, N}(t) m_{N}(0) E_{\ell, N}^{\top}(t),
$$

Proof: Define $m_{\infty}(0)$ as the semi-infinite matrix formed by putting $m_{N}(0)$ in the upper-left corner and setting all other entries equal to 0 and let $\Lambda_{\infty}$ be the semi-infinite shift matrix. Then the solution to the differential equations

$$
\frac{\partial m_{\infty}}{\partial t_{k}}=\Lambda_{\infty}^{k} m_{\infty}+m_{\infty} \Lambda_{\infty}^{\top k}
$$

is given by

$$
m_{\infty}(t)=e^{\sum_{1}^{\infty} t_{k} \Lambda_{\infty}^{k}} m_{\infty}(0) e^{\sum_{1}^{\infty} t_{k} \Lambda_{\infty}^{\top k}} .
$$

Result (2.0.1) follows from the Taylor expansion

$$
e^{\sum_{1}^{\infty} t_{k} \Lambda_{\infty}^{k}}=\sum_{k=0}^{\infty} \mathbf{s}_{k}(t) \Lambda_{\infty}^{k},
$$

which is an upper-triangular semi-infinite matrix, and considering only the upper-left $\ell \times \ell$ block. Each upper-left $\ell \times \ell$ block of $m_{\infty}(t)$ for $\ell \leq N$, equals

$$
\begin{aligned}
m_{\ell}(t) & =E_{\ell, \infty}(t) m_{\infty}(0) E_{\ell, \infty}^{\top}(t) \\
& =E_{\ell, N}(t) m_{N}(0) E_{\ell, N}^{\top}(t)
\end{aligned}
$$

from which (2.0.3) follows, and (2.0.2) setting $\ell=N$.

Remark: The flow (2.0.4) maintains the finite upper-left hand corner of $m_{\infty}$ and on that locus it is equivalent to the finite flow (2.0.1). Therefore, the whole semi-infinite theory can be applied to this case. It is possible to give a proof of Theorem 2.1 purely within finite matrices. 
Theorem 2.2 Consider the commuting equations on the $N \times N$ matrix in

$$
\frac{\partial m_{N}}{\partial t_{i}}=\Lambda^{i} m_{N}+m_{N} \Lambda^{i}
$$

with skew-symmetric initial condition $m_{N}(s)$ and its "skew-Borel decomposition"

$$
m_{N}=Q^{-1} J Q^{-1 \top}, \text { for } Q \in G_{\mathfrak{k}}
$$

When $N$ is odd, we further impose the differential equations for the last entry $Q_{N N}$ of $Q$ :

$$
\frac{\partial Q_{N N}}{\partial t_{i}}=-\frac{1}{2} Q_{N, N-i} .
$$

Then for arbitrary $N>0$ the matrix $Q$ evolves according to the equations

$$
\frac{\partial Q}{\partial t_{i}} Q^{-1}=-\pi_{\mathfrak{k}}\left(Q \Lambda^{i} Q^{-1}\right)
$$

and the matrix $L:=Q \Lambda Q^{-1}$ provides a solution to the Lax pair

$$
\frac{\partial L}{\partial t_{i}}=\left[-\pi_{\mathfrak{k}} L^{i}, L\right]=\left[\pi_{\mathfrak{n}} L^{i}, L\right]
$$

Proof: For a matrix $A$, consider the projections

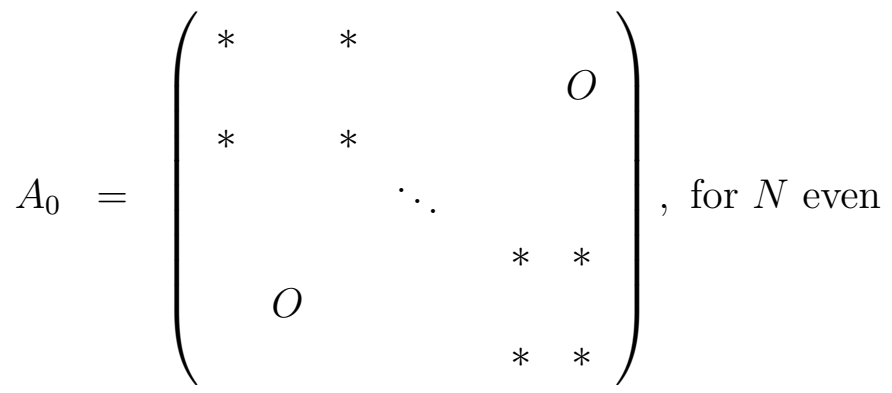

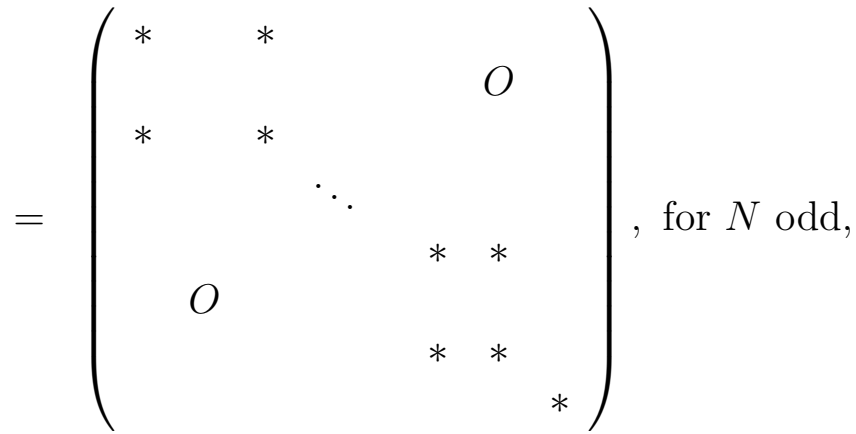


and

$$
\begin{aligned}
& A_{00}=A_{0} \text {, for } N \text { even }
\end{aligned}
$$

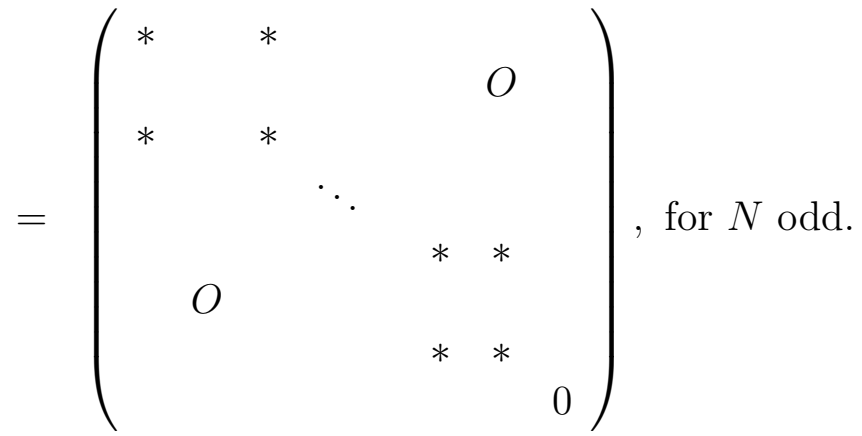

The main point is to prove that

$$
\begin{aligned}
0 & =\frac{\partial Q}{\partial t_{i}} Q^{-1}+\pi_{\mathfrak{k}} L^{i} \\
& =\frac{\partial Q}{\partial t_{i}} Q^{-1}+\left(L_{-}^{i}-J\left(L_{+}^{i}\right)^{\top} J\right)+\frac{1}{2}\left(L_{0}^{i}-J\left(L_{0}^{i}\right)^{\top} J\right) \\
& =: A .
\end{aligned}
$$

Also define

$$
\left(L^{i}+\frac{\partial Q}{\partial t_{i}} Q^{-1}\right)-J\left(L^{i}+\frac{\partial Q}{\partial t_{i}} Q^{-1}\right)^{\top} J=: B
$$

we have, setting $\cdot=\frac{\partial}{\partial t_{i}}$,

$$
\begin{gathered}
0=Q\left(\Lambda^{i} m+m \Lambda^{\top i}-\frac{\partial m}{\partial t_{i}}\right) Q^{\top} \\
=\left(Q \Lambda^{i} Q^{-1}\right) J+J Q^{-1 \top} \Lambda^{\top i} Q^{\top}+\left(\dot{Q} Q^{-1}\right) J+J Q^{-1 \top} \dot{Q}^{\top} \\
=\left(L^{i}+\dot{Q} Q^{-1}\right) J+J\left(L^{i}+\dot{Q} Q^{-1}\right)^{\top} . \\
{ }^{5} L_{+}^{i}:=\left(L^{i}\right)_{+} \text {and } L_{0}^{i}:=\left(L^{i}\right)_{0} .
\end{gathered}
$$


Hence

$$
\begin{aligned}
0 & =\left(Q\left(\Lambda^{i} m+m \Lambda^{\top i}-\frac{\partial m}{\partial t_{i}}\right) Q^{\top}\right)_{-, 00} \\
& =\left(\left(\left(L^{i}+\dot{Q} Q^{-1}\right)-J\left(L^{i}+\dot{Q} Q^{-1}\right)^{\top} J\right) J\right)_{-, 00} \\
& =\left(\left(L^{i}+\dot{Q} Q^{-1}\right)-J\left(L^{i}+\dot{Q} Q^{-1}\right)^{\top} J\right)_{-, 00} J \\
& =B_{-, o o} J .
\end{aligned}
$$

Therefore

$$
0=B_{-, 00} J^{2}= \begin{cases}B_{-, 0}, & \text { for } N \text { even } \\
B_{-, 00}\left(\begin{array}{cc}
I_{N-1} & O \\
O & 0
\end{array}\right) & \text { for } N \text { odd }\end{cases}
$$

and so

$$
B_{-}=0 \quad \text { and } \quad B_{00}=0 .
$$

But

$$
\begin{aligned}
B_{-} & =\left(L^{i}+\dot{Q} Q^{-1}-J\left(L_{+}^{i}\right)^{\top} J\right)_{-} \\
& =\left(\dot{Q} Q^{-1}\right)_{-}+\left(\left(L^{i}\right)_{-}-J\left(L_{+}^{i}\right)^{\top} J\right) \\
& =A_{-}
\end{aligned}
$$

and

$$
\begin{aligned}
B_{00} & =2\left(\dot{Q} Q^{-1}\right)_{00}+\left(L^{i}-J\left(L^{i}\right)^{\top} J\right)_{00} \\
& =2 A_{00} .
\end{aligned}
$$

Then, by (2.0.12) and (2.0.13),

$$
0=B_{-}+\frac{1}{2} B_{00}=A_{-}+A_{00}=A_{-}+A_{00}+A_{+}, \quad \text { since } A_{+}=0 .
$$

Therefore, when $\underline{N \text { is even, }} A=0$ and the proof is finished. When $\underline{N \text { is odd, }}$ we have

$$
{ }^{6} A_{-, 00}=A_{-}+A_{00} .
$$




$$
A=0, \text { except for the }(N, N) \text {-entry. }
$$

But the $(N, N)$ th entry of $L^{i}$ is given by, since $Q$ is lower-triangular,

$$
\left(L^{i}\right)_{N N}=\left(Q \Lambda^{i} Q^{-1}\right)_{N N}=\frac{Q_{N, N-i}}{Q_{N N}}
$$

and thus we have, using the fact that the $(N, N)$ th entry of $J\left(L^{i}\right)_{0} J$ vanishes,

$$
\begin{aligned}
A_{N N} & =\frac{\partial}{\partial t_{i}} \log Q_{N N}+\frac{1}{2}\left(L^{i}\right)_{N N} \\
& =\frac{1}{Q_{N N}}\left(\frac{\partial Q_{N N}}{\partial t_{i}}+\frac{1}{2} Q_{N, N-i}\right), \\
& =0, \text { by the assumption (2.0.8), }
\end{aligned}
$$

thus ending the proof of Theorem 2.2.

\section{The solution to the Pfaff lattice with anti- diagonal skew-symmetric initial condition}

Consider the equations

$$
\frac{\partial m_{N}}{\partial t_{i}}=\Lambda^{i} m_{N}+m_{N} \Lambda^{\top i}
$$


with initial condition,

$$
\begin{aligned}
& m_{N}(0)=\left(\begin{array}{cccc} 
& & & \\
& & & b^{\frac{N-2}{2}} \\
& & b_{0} & \\
-b_{\frac{N-2}{2}} & & &
\end{array}\right) \text {, for } N \text { even, }
\end{aligned}
$$

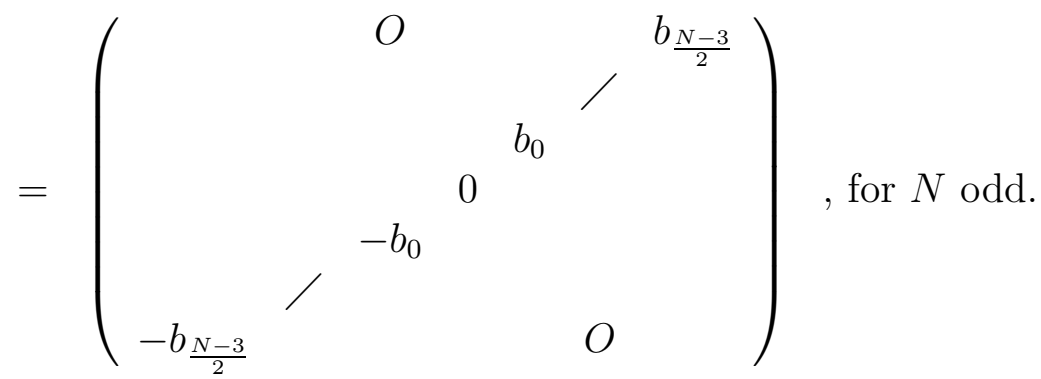

Proposition 3.1 The system of equations (3.0.1), with initial condition (3.0.2) have for solution the matrix $m_{N}(t)$, with entries, for $0 \leq \ell<k \leq N$,

$$
\begin{aligned}
\mu_{\ell, k}(t)=- & \sum_{j=0}^{\left[\frac{N-2}{2}\right]-k} \mathbf{s}_{j} \mathbf{s}_{N-\ell-k-j-1}\left(b_{\left[\frac{N-2}{2}\right]-k-j}-b_{\left[\frac{N-2}{2}\right]-\ell-j}\right) \\
& -\sum_{\left[\frac{N-2}{2}\right]-k+1}^{\left[\frac{N-2}{2}\right]-\ell} \mathbf{s}_{j} \mathbf{s}_{N-\ell-k-j-1}\left(-b_{\left[\frac{N-2}{2}\right]-\ell-j}\right) .
\end{aligned}
$$

In particular

$$
\begin{aligned}
\mu_{01}(t) & =\sum_{i=0}^{\frac{N-2}{2}} b_{i} \mathbf{s}_{\frac{N-2}{2}+i, \frac{N-2}{2}-i}(t), \quad \text { for } N \text { even } \\
& =\sum_{i=0}^{\frac{N-3}{2}} b_{i} \mathbf{s}_{\frac{N-1}{2}+i, \frac{N-3}{2}-i}(t), \quad \text { for } N \text { odd }
\end{aligned}
$$


Proof: Equation (3.0.3) is established by explicit computation of

$$
\begin{aligned}
m_{N}(t) & =E_{N, N}(t) m_{N}(0) E_{N, N}(t)^{\top} \\
& =\left(\sum_{i, j=0}^{N-\ell-1} \mathbf{s}_{i}(t) \mu_{i+\ell, j+k}(0) \mathbf{s}_{j}(t)\right)_{0 \leq \ell, k \leq N-1} .
\end{aligned}
$$

From (3.0.3), one computes, for $N$ even,

$$
\begin{aligned}
\mu_{01}(t)= & \mathbf{s}_{0} \mathbf{s}_{N-2}\left(b_{\frac{N}{2}-1}-b_{\frac{N}{2}-2}\right)+\mathbf{s}_{1} \mathbf{s}_{N-3}\left(b_{\frac{N}{2}-2}-b_{\frac{N}{2}-3}\right)+ \\
& \cdots+\mathbf{s}_{\frac{N}{2}-2} \mathbf{s}_{\frac{N}{2}}\left(b_{1}-b_{0}\right)+\left(\mathbf{s}_{\frac{N}{2}-1}\right)^{2} b_{0} \\
= & \sum_{i=0}^{\frac{N}{2}-1} b_{i}\left(\mathbf{s}_{\frac{N}{2}-1-i} \mathbf{s}_{\frac{N}{2}-1+i}-\mathbf{s}_{\frac{N}{2}-2-i} \mathbf{s}_{\frac{N}{2}+i}\right) \\
= & \sum_{i=0}^{\frac{N}{2}-1} b_{i} \mathbf{s}_{\frac{N}{2}-1+i, \frac{N}{2}-1-i}(t)
\end{aligned}
$$

and for $N$ odd,

$$
\begin{aligned}
\mu_{01}(t)= & \mathbf{s}_{0} \mathbf{s}_{N-2}\left(b_{\frac{N-3}{2}}-b_{\frac{N-5}{2}}\right)+\mathbf{s}_{1} \mathbf{s}_{N-3}\left(b_{\frac{N-5}{2}}-b_{\frac{N-7}{2}}\right)+ \\
& \ldots+\mathbf{s}_{\frac{N-5}{2}} \mathbf{s}_{\frac{N+1}{2}}\left(b_{1}-b_{0}\right)+\mathbf{s}_{\frac{N-3}{2}} \mathbf{s}_{\frac{N-1}{2}} b_{0} \\
= & \sum_{i=0}^{\frac{N-3}{2}} b_{i} \mathbf{s}_{\frac{N-1}{2}+i, \frac{N-3}{2}-i}(t),
\end{aligned}
$$

ending the proof of Proposition 3.1.

Definel

$$
\begin{aligned}
m_{N}(0 ; z) & :=m_{N}(0), \quad \text { for } N \text { even, } \\
m_{N}(0 ; z):= & m_{N}(0)+z^{2} \varepsilon_{\frac{N+1}{2}, \frac{N+1}{2}}, \quad \text { for } N \text { odd }, \\
O & b_{\frac{N-3}{2}} \\
= & \left(\begin{array}{r}
b_{0}^{2} \\
-b_{\frac{N-3}{2}}
\end{array}\right)
\end{aligned}
$$

\footnotetext{
${ }^{7} \varepsilon_{i, j}$ denotes the matrix with all zero entries, except for a 1 at the $(i, j)$ th entry.
} 


\section{Proposition 3.2}

$$
\begin{aligned}
& \operatorname{det}^{1 / 2}\left(E_{\ell, N}(t) m_{N}(0 ; z) E_{\ell, N}^{\top}(t)\right) \\
& =z^{\eta(N, \ell)} \sum_{\lambda \in \mathbb{Y}_{\frac{\ell(N-\ell)}{2}}^{(\ell)}}\left(\prod_{1}^{[\ell / 2]} b_{\lambda_{i}-i+\ell-\left[\frac{N+1}{2}\right]}\right) \mathbf{s}_{\lambda_{1} \geq \ldots \geq \lambda_{\ell}}(t) .
\end{aligned}
$$

with

$$
\eta(N, \ell)= \begin{cases}1, & \text { for } N \text { and } \ell \text { odd } \\ 0, & \text { otherwise. }\end{cases}
$$

Lemma 3.3 Consider an arbitrary $N \times N$ matrix $A=\left(A_{i j}\right)_{1 \leq i, j \leq N}$, with $r=\left[\frac{N}{2}\right]$ and $A_{\ell}:=\left(A_{i j}\right)_{\substack{1 \leq i \leq \ell \leq N \\ 1 \leq j \leq N}}$ and consider the anti-diagonal matrix

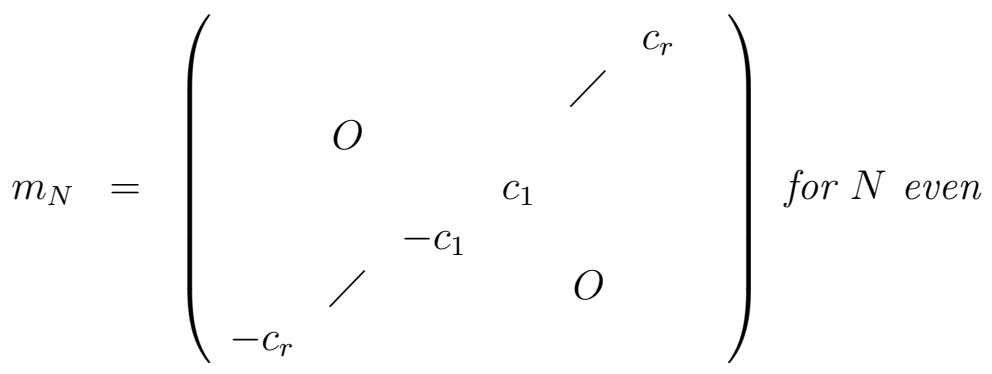

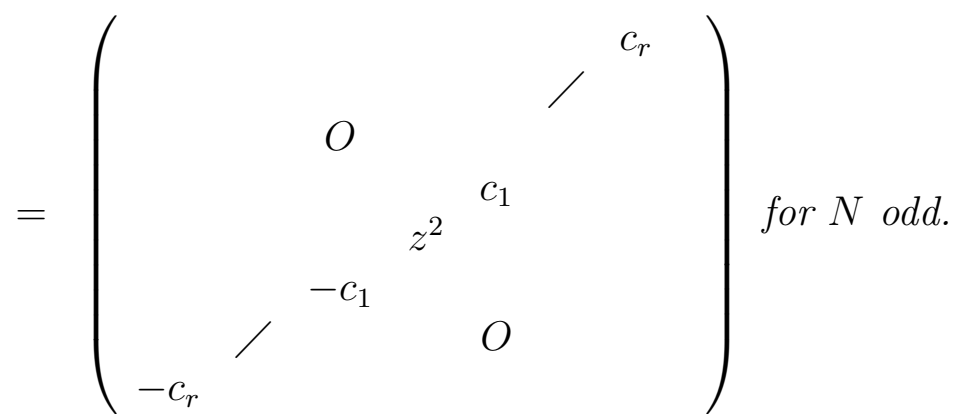

Setting

$$
m_{\ell}^{A}(z):=A_{\ell} m_{N}(z) A_{\ell}^{\top}
$$

and

\footnotetext{
${ }^{8} B_{\left(j_{1}, \ldots, j_{n}\right)}$ denotes the matrix formed with the columns $j_{1}, \ldots, j_{n}$ of $B$
} 


$$
\begin{aligned}
& P_{N, \ell}=\sum_{1 \leq i_{1} \leq \ldots \leq i_{[\ell / 2]} \leq r} c_{i_{1}} \ldots c_{i_{[\ell / 2]}} \\
& \times\left\{\begin{array}{l}
\operatorname{det}\left(A_{\ell}\right)_{\left(r-i_{[\ell / 2]}+1, \ldots, r-i_{1}+1, r+i_{1}, \ldots, r+i_{[\ell / 2]}\right)} \quad \text { for } N \text { even, } \ell \text { even } \\
\operatorname{det}\left(A_{\ell}\right)_{\left(r-i_{[\ell / 2]}+1, \ldots, r-i_{1}+1, r+i_{1}+1, \ldots, r+i_{[\ell / 2]}+1\right)} \quad \text { for } N \text { odd, } \ell \text { even } \\
\operatorname{det}\left(A_{\ell}\right)_{\left(r-i_{[\ell / 2]}+1, \ldots, r-i_{1}+1, r+1, r+i_{1}+1, \ldots, r+i_{[\ell / 2]}+1\right)} \quad \text { for } N \text { odd, } \ell \text { odd }
\end{array}\right.
\end{aligned}
$$

we have

$$
\operatorname{det} m_{\ell}^{A}=\left\{\begin{array}{cl}
0 & \text { for } N \text { even, } \ell \text { odd } \\
\left(p f m_{\ell}^{A}\right)^{2}=\left(P_{N, \ell}\right)^{2} & \text { for } N \text { even, } \ell \text { even } \\
z^{2} P_{N, \ell}^{2} & \text { for } N \text { odd, } \ell \text { odd } \\
\left(p f m_{\ell}^{A}(0)\right)^{2}=\left(P_{N, \ell}\right)^{2} & \text { for } N \text { odd, } \ell \text { even }
\end{array}\right.
$$

Proof: Let $w_{i} \in \mathbb{C}^{\ell}$ be the columns of $A_{\ell}$

$$
A_{\ell}=\left[w_{0}, w_{1}, \ldots, w_{2 r}\right]
$$

and observe

$$
\begin{aligned}
m_{\ell}^{A}(z)=A_{\ell} m_{N}(z) A_{\ell}^{\top} & =A_{\ell}\left(z^{2} \varepsilon_{r+1, r+1}+m_{N}(0)\right) A_{\ell}^{\top} \\
& =z^{2} w_{r} \otimes w_{r}+m_{\ell}^{A}(0) .
\end{aligned}
$$

Let $U$ be a $\ell \times \ell$ matrix, rational in the $a_{i j}$, such that

$$
U w_{r}=\alpha e_{1}, \quad \operatorname{det} U=1 .
$$

Then, using $U(x \otimes y) V=(U x) \otimes\left(V^{\top} y\right)$ and setting $M:=U m_{\ell}^{A}(0) U^{\top}$, which is skew-symmetric, we find 


$$
\begin{aligned}
\operatorname{det} m_{\ell}^{A}(z) & =\operatorname{det} U m_{\ell}^{A}(z) U^{\top} \\
& =\operatorname{det}\left(z^{2} U\left(w_{r} \otimes w_{r}\right) U^{\top}+U m_{\ell}^{A}(0) U^{\top}\right) \\
& =\operatorname{det}\left(z^{2} \alpha^{2} e_{1} \otimes e_{1}+U m_{\ell}^{A}(0) U^{\top}\right) \\
& =\operatorname{det}\left(\begin{array}{c|cccc}
(z \alpha)^{2} & M_{12} & M_{13} & \ldots & M_{1 \ell} \\
\hline-M_{12} & 0 & M_{23} & \ldots & M_{2 \ell} \\
-M_{13} & -M_{23} & & \\
\vdots & \vdots & & \vdots \\
-M_{1 \ell} & -M_{2 \ell} & \ldots & & 0
\end{array}\right) \\
& =(z \alpha)^{2} \operatorname{det}\left(M_{i j}\right)_{2 \leq i, j \leq \ell}+\operatorname{det}\left(M_{i j}\right)_{1 \leq i, j \leq \ell},
\end{aligned}
$$

with $M_{i j}=-M_{j i}$. Therefore

$$
\begin{aligned}
\operatorname{det} m_{\ell}^{A}(z) & =\operatorname{det} m_{\ell}^{A}(0)=\left(p f m_{\ell}^{A}(0)\right)^{2}, & & \text { for } \ell \text { even } \\
& =(z \alpha)^{2} \operatorname{det}\left(M_{i j}\right)_{2 \leq i, j \leq \ell}=\left(z \alpha p f\left(M_{i j}\right)_{2 \leq i, j \leq \ell}\right)^{2}, & & \text { for } \ell \text { odd },
\end{aligned}
$$

the latter being the square of a polynomial in $z$, the $c_{i}$ and the entries of the matrix $A$. 
Using the Cauchy-Bonnet formula twice, one computes, say, for $N$ and $\ell$ odd,

$$
\begin{aligned}
& \operatorname{det} m_{\ell}^{A}(z)=\operatorname{det} A_{\ell} m_{N}(z) A_{\ell}^{\top} \\
& =\sum_{1 \leq \alpha_{1}<\ldots<\alpha_{\ell} \leq N} \operatorname{det}\left(\left(A_{\ell}\right)_{i, \alpha_{j}}\right)_{1 \leq i, j \leq \ell} \operatorname{det}\left(\left(A_{\ell} m^{\top}\right)_{i, \alpha_{j}}\right)_{1 \leq i, j \leq \ell} \\
& =\sum_{\substack{1 \leq \alpha_{1}<\ldots<\alpha_{\ell} \leq N \\
1 \leq \beta_{1}<<<\beta_{\ell} \leq N \\
\alpha_{i}+\beta_{\ell}-i+1=N+1}} \operatorname{det}\left(\left(A_{\ell}\right)_{i, \alpha_{j}}\right)_{1 \leq i, j \leq \ell} \operatorname{det}\left(\left(A_{\ell}\right)_{i, \beta_{j}}\right)_{1 \leq i, j \leq \ell} \operatorname{det}\left(\left(m^{\top}\right)_{\beta_{i}, \alpha_{j}}\right)_{1 \leq i, j \leq \ell} \\
& =\sum_{\substack{1 \leq \alpha_{1}<\ldots<\alpha_{\ell} \leq N \\
1 \leq \beta_{1}<\ldots<\beta_{\ell} \leq N \\
\alpha_{i}+\beta \ell-i+N \\
\text { for } 1 \leq i \leq \ell}} \operatorname{det}\left(\left(A_{\ell}\right)_{i, \alpha_{j}}\right)_{1 \leq i, j \leq \ell} \operatorname{det}\left(\left(A_{\ell}\right)_{i, \beta_{j}}\right)_{1 \leq i, j \leq \ell} \operatorname{det}\left(m_{\alpha_{i}, \beta_{j}}\right)_{1 \leq i, j \leq \ell} \\
& =\left(\sum_{\substack{1 \leq \alpha_{1}<\ldots<\alpha_{\ell} \leq N \\
1 \leq \beta_{1}<\ldots<\beta_{\ell} \leq N}}+\sum_{\substack{1 \leq \alpha_{1}<\ldots<\alpha_{\ell} \leq N \\
1 \leq \beta_{1}<\ldots<\beta_{\ell} \leq N}}\right) \operatorname{det}\left(\left(A_{\ell}\right)_{i, \alpha_{j}}\right)_{1 \leq i, j \leq \ell} \operatorname{det}\left(\left(A_{\ell}\right)_{i, \beta_{j}}\right)_{1 \leq i, j \leq \ell} \\
& \begin{array}{cc}
\left(\alpha_{1}, \ldots, \alpha_{\ell}\right)=\left(\beta_{1}, \ldots, \beta_{\ell}\right) & \left(\alpha_{1}, \ldots, \alpha_{\ell}\right) \neq\left(\beta_{1}, \ldots, \beta_{\ell}\right) \\
\alpha_{i}+\beta_{\ell-i+1=N+1} & \alpha_{i}+\beta_{\ell-i+1}=N+1 \\
\text { for } 1 \leq i \leq \ell & \text { for } 1 \leq i \leq \ell
\end{array} \\
& \times \operatorname{det}\left(m_{\alpha_{i}, \beta_{j}}\right)_{1 \leq i, j \leq \ell} \\
& \stackrel{*}{=} z^{2} \sum_{1 \leq \alpha_{1}<\ldots<\alpha_{\frac{\ell-1}{2}<\frac{N+1}{2}}} c_{\frac{N+1}{2}-\alpha_{1}}^{2} \ldots c_{\frac{N+1}{2}-\alpha_{\frac{\ell-1}{2}}}^{2} \operatorname{det}^{2}\left(\left(A_{\ell}\right)_{i, \alpha_{j}}\right)_{1 \leq i, j \leq \ell}+\ldots \\
& \begin{array}{l}
\alpha_{\ell+1}+i \\
2+\alpha \frac{\ell+1}{2}-i \\
\text { for } 0 \leq i \leq \frac{\ell-1}{2}
\end{array} \\
& =\left(z \sum_{\substack{1 \leq \alpha_{1}<\ldots<\alpha_{\frac{\ell-1}{1}}<\frac{N+1}{2} \\
\alpha_{\frac{\ell+1}{2}+i}+\alpha \frac{\ell+1}{2}-i}} c_{\frac{N+1}{2}-\alpha_{1}} \ldots c_{\frac{N+1}{2}-\alpha_{\frac{\ell-1}{2}}} \operatorname{det}\left(\left(A_{\ell}\right)_{i, \alpha_{j}}\right)_{1 \leq i, j \leq \ell}\right)^{2} \operatorname{using}(\text { 3.0.7) }
\end{aligned}
$$

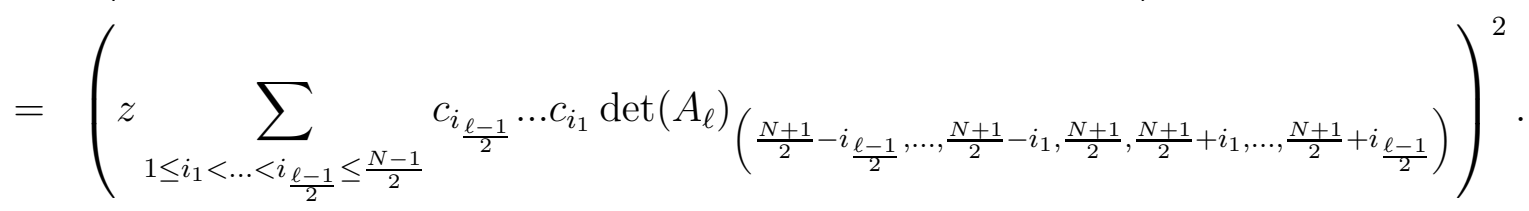


In $\stackrel{*}{=}$ we have used the fact that

$$
\left.\begin{array}{c}
\left(\alpha_{1}, \ldots, \alpha_{\ell}\right)=\left(\beta_{1}, \ldots, \beta_{\ell}\right) \\
\alpha_{i}+\beta_{\ell-i+1}=N+1
\end{array}\right\} \Longleftrightarrow\left\{\begin{array}{c}
\alpha_{\frac{\ell+1}{2}+i}+\alpha_{\frac{\ell+1}{2}-i}=N+1 \\
\text { for } 0 \leq i \leq \frac{\ell-1}{2} \\
\beta_{\ell-i+1}=N+1-\alpha_{i} .
\end{array}\right.
$$

Indeed, for $\underline{N \text { odd }}$ consider sequences $\alpha_{i}$ symmetric about

$$
\alpha_{\frac{\ell+1}{2}}=\frac{N+1}{2}
$$

i.e.

$$
\alpha_{\frac{\ell+1}{2}+i}+\alpha_{\frac{\ell+1}{2}-i}=N+1, \text { for } 0 \leq i \leq \frac{\ell-1}{2} .
$$

Then, using (3.0.8) and (3.0.10

$$
\beta_{\frac{\ell+1}{2}-i}=N+1-\alpha_{\frac{\ell+1}{2}+i}=\alpha_{\frac{\ell+1}{2}-i},
$$

thus implying

$$
\left(\alpha_{1}, \ldots, \alpha_{\ell}\right)=\left(\beta_{1}, \ldots, \beta_{\ell}\right) .
$$

Vice versa, the latter implies (3.0.8) and thus (3.0.9). This establishes Lemma 3.3 for the case $N$ and $\ell$ odd; for the other cases, one proceeds in a similar fashion.

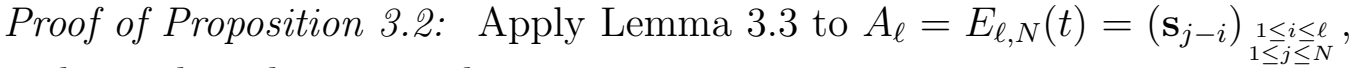
with $1 \leq k_{1}<k_{2}<\ldots<k_{\ell}$ :

$$
\begin{aligned}
\operatorname{det}\left(A_{\ell}\right)_{k_{1}, \ldots, k_{\ell}} & =\operatorname{det}\left(\begin{array}{cccc}
\mathbf{s}_{k_{1}-1} & \ldots & \mathbf{s}_{k_{\ell-1}-1} & \mathbf{s}_{k_{\ell}-1} \\
\vdots & & \vdots & \vdots \\
\mathbf{s}_{k_{1}-\ell} & \ldots & \mathbf{s}_{k_{\ell-1}-\ell} & \mathbf{s}_{k_{\ell}-\ell}
\end{array}\right) \\
& =\operatorname{det}\left(\begin{array}{cccc}
\mathbf{s}_{k_{\ell}-\ell} & \mathbf{s}_{k_{\ell}-\ell+1} & \ldots & \mathbf{s}_{k_{\ell}-1} \\
\mathbf{s}_{k_{\ell-1}-\ell} & \mathbf{s}_{k_{\ell-1}-\ell+1} & \ldots & \mathbf{s}_{k_{\ell-1}-1} \\
\vdots & & & \vdots \\
\mathbf{s}_{k_{1}-\ell} & & \ldots & \mathbf{s}_{k_{1}-1}
\end{array}\right) \\
& =\mathbf{s}_{k_{\ell}-\ell, k_{\ell-1}-\ell+1, \ldots, k_{1}-\ell+(\ell-1)} \\
& =\mathbf{s}_{\lambda_{1} \geq \ldots \geq \lambda_{\ell}} \\
& =\mathbf{s}_{\lambda}
\end{aligned}
$$


where

$$
\lambda_{i}=k_{\ell-i+1}-\ell+i-1 \text {, for } 1 \leq i \leq \ell .
$$

In order to apply Lemma 3.3, the $k_{i}$ inherent in formula (3.0.6) must be as in formula (6.0.4); i.e., setting $r=[N / 2]$, the $k_{j}$ 's must satisfy

$$
k_{j}=\left[\frac{N}{2}\right]-i_{[\ell / 2]-j+1}+1=N+1-k_{\ell-j+1}, \quad \text { for } 1 \leq j \leq\left[\frac{\ell+1}{2}\right]
$$

and thus

$$
\begin{aligned}
i_{[\ell / 2]+1-j}-1 & =k_{\ell+1-j}-\left[\frac{N+1}{2}\right]-1 \\
& =\lambda_{j}+\ell-j-\left[\frac{N+1}{2}\right] .
\end{aligned}
$$

Therefore, formula (3.0.6) can be applied with

$$
c_{i_{[\ell / 2]-j+1}}=b_{\lambda_{j}+\ell-j-[(N+1) / 2]}, \quad \text { for } 1 \leq j \leq\left[\frac{\ell}{2}\right] .
$$

From (3.0.12) and (3.0.13), it follows that

$$
\lambda_{i}+\lambda_{\ell+1-i}=k_{\ell+1-i}+k_{i}-\ell-1=N+1-\ell-1=N-\ell,
$$

showing that

$$
\lambda \in \mathbb{Y}_{\frac{\ell(N-\ell)}{2}}^{(\ell)}
$$

establishing Proposition 3.2.

\section{Proof of Theorem 1.1}

Using the standard notation for the partition $1^{j}=\overbrace{(1, \ldots, 1)}^{j}$, we state

Lemma 4.1 For

$$
\left.\begin{array}{l}
\mathbf{s}_{i}(-\tilde{\partial}) \mathbf{s}_{1^{j}}(t) \\
\left(-\frac{\partial}{\partial t_{i}}\right) \mathbf{s}_{1^{j}}(t)
\end{array}\right\}=(-1)^{i} \mathbf{s}_{1^{j-i}}(t)
$$


Proof: Using the usual inner-product between symmetric functions, we have

$$
\begin{aligned}
\mathbf{s}_{i}(\tilde{\partial}) \mathbf{s}_{j}(t) & =\left\langle\mathbf{s}_{i}(t+u) \cdot 1, \mathbf{s}_{j}(t+u)\right\rangle \\
& =\left\langle\mathbf{s}_{j}(t+u), \mathbf{s}_{i}(t+u) \cdot 1\right\rangle \\
& =\left\langle\mathbf{s}_{j-i}(t+u), 1\right\rangle \\
& =\left\langle 1, \mathbf{s}_{j-i}(t+u)\right\rangle \\
& =\left.\mathbf{s}_{j-i}(t+u)\right|_{u=0} \\
& =\mathbf{s}_{j-i}(t)
\end{aligned}
$$

and so, changing $t \mapsto-t$,

$$
\mathbf{s}_{i}(-\tilde{\partial}) \mathbf{s}_{j}(-t)=\mathbf{s}_{j-i}(-t),
$$

from which this first relation follows upon noticing that

$$
\mathbf{s}_{j}(-t)=(-1)^{j} \mathbf{s}_{1^{j}}(t) .
$$

This last relation (4.0.2) also leads to the second identity (4.0.1), using $\left(\partial / \partial t_{i}\right) \mathbf{s}_{j}(t)=\mathbf{s}_{j-i}(t)$.

Proof of Theorem 1.1: By Proposition 2.1, the equations for the $N \times N$ matrix $m_{N}$

$$
\frac{\partial m_{N}}{\partial t_{k}}=\Lambda^{k} m_{N}+m_{N} \Lambda^{k},
$$

with skew-symmetric initial condition $m_{N}(0)$ has the following solution

$$
m_{N}(t)=E_{\ell, N} m_{N}(0) E_{\ell, N}^{\top}(t),
$$

which remains skew-symmetric in time. Define a $t$-dependent skew-inner product such that $\left\langle y^{i}, z^{j}\right\rangle_{t}=m_{i j}(t)$, i.e. f,

$$
\left\langle\chi_{N}(y) \chi(z)^{\top}\right\rangle=m_{N}(t) .
$$

Performing the skew Borel decomposition

$$
\frac{m_{N}(t)=Q^{-1}(t) J Q^{-1 \top}, \quad \text { with } Q(t) \in G_{k}}{{ }^{9} \chi(y):=\left(1, y, y^{2}, \ldots\right)^{\top} .}
$$


is tantamount to the process of finding a finite set of skew-orthonormal polynomials; that is, satisfying

$$
\left(\left\langle q_{i}(t ; z), q_{j}(t ; z)\right\rangle\right)_{1 \leq i, j \leq N}=J
$$

Indeed, the polynomials $q_{i}(t ; z)$ in $z$, depending on $t$,

$$
\left(\begin{array}{c}
q_{0} \\
q_{1} \\
\vdots \\
q_{N-1}
\end{array}\right)=Q\left(\begin{array}{c}
1 \\
z \\
\vdots \\
z^{N-1}
\end{array}\right)
$$

satisfy

$$
\begin{aligned}
\left(\left\langle q_{i}(t ; y), q_{i}(t ; z)\right\rangle\right)_{0 \leq i, j \leq N-1} & =\left\langle Q(t) \chi_{N}(y), Q(t) \chi_{N}(z)\right\rangle \\
& =\left\langle Q(t) \chi_{N}(y) \chi_{N}(z) Q^{\top}(t)\right\rangle \\
& =Q(t)\left\langle\chi_{N}(y) \chi_{N}(z)\right\rangle Q^{\top}(t) \\
& =Q(t) m_{N}(t) Q^{\top}(t) \\
& =J .
\end{aligned}
$$

According to [2], the skew-orthogonal polynomials are related to the $\tau$ functions $\left(\tau_{0}=1, \tau_{N}=c\right)$

$$
\tau_{\ell}(t)=p f m_{\ell}(t)
$$

as follows

$$
\begin{aligned}
q_{2 n} & =\frac{z^{2 n}}{\sqrt{\tau_{2 n} \tau_{2 n+2}}} \tau_{2 n}\left(t-\left[z^{-1}\right]\right) \\
q_{2 n+1} & =\frac{z^{2 n}}{\sqrt{\tau_{2 n} \tau_{2 n+2}}}\left(z+\frac{\partial}{\partial t_{1}}\right) \tau_{2 n}\left(t-\left[z^{-1}\right]\right), \quad 0 \leq 2 n \leq N-2 .
\end{aligned}
$$

This ends the proof of Theorem 1.1 for $N$ even. However for odd $N$, we must verify condition (2.0.8) of Theorem 2.2. This requires knowing $q_{N-1}(t ; z)$ explicitly. For later purposes we shall also need $q_{N-1}(t ; z)$ for even $N$. 
For $N$ even, $q_{N-1}$ takes on the following form

$$
q_{N-1}(t ; z)=\frac{z^{N-2}}{\sqrt{\tau_{N-2} \tau_{N}}}\left(z+\frac{\partial}{\partial t_{1}}\right) \tau_{N-2}\left(t-\left[z^{-1}\right]\right)
$$

with (using Proposition 3.2)

$$
\tau_{N-2}(t)=\sum_{\lambda \in \mathbb{Y}_{N-2}^{(N-2)}}\left(\prod_{1}^{\frac{N-2}{2}} b_{\lambda_{i}-i+\frac{N}{2}-2}\right) \mathbf{s}_{\lambda}(t),
$$

where

$$
\mathbb{Y}_{N-2}^{(N-2)}=\left\{1^{N-2},\left(2,1^{N-4}\right),\left(2^{2}, 1^{N-6}\right), \ldots,\left(2^{i}, 1^{N-2 i-2}\right), \ldots\right\}
$$

For $N$ odd,$q_{N-1}$ has the form

$$
q_{N-1}(t ; z)=\frac{z^{N-1}}{\sqrt{\tau_{N-1}}} \tau_{N-1}\left(t-\left[z^{-1}\right]\right)
$$

with

$$
\tau_{N-1}(t)=b_{0} \ldots b_{\frac{N-3}{2}} \mathbf{s}\left(1^{\frac{N-1}{2}}\right)(t) .
$$

Indeed, observe that the set of partitions

$$
\begin{aligned}
\left.\mathbb{Y}_{\frac{\ell(N-\ell)}{2}}^{\ell}\right|_{\ell=N-1}=\mathbb{Y}_{\frac{N-1}{2}}^{(N-1)} & =\left\{\begin{array}{c}
\left(\lambda_{1}, \ldots, \lambda_{N-1}\right) \in \mathbb{Y}_{\frac{N-1}{2}} \\
\text { with } \lambda_{i}+\lambda_{\ell+1-i}=1
\end{array}\right\} \\
& =\left\{1^{\frac{N-1}{2}}\right\}
\end{aligned}
$$

consists of one element $1^{\frac{N-1}{2}}$. Therefore, setting $\lambda_{i}=1$ for $1 \leq i \leq \frac{N-1}{2}$ one finds, again by Proposition 3.2,

$$
\tau_{N-1}(t)=b_{0} \ldots b_{\frac{N-3}{2}} \mathbf{s}\left({ }_{1}^{\frac{N-1}{2}}\right)(t) .
$$


The last row of $\tilde{Q}$ is given by:

$$
\begin{aligned}
\sum_{0}^{N-1} \tilde{Q}_{N, j+1} z^{j} & =z^{N-1} \tau_{N-1}\left(t-\left[z^{-1}\right]\right) \\
& =\sum_{i=0}^{N-1} \mathbf{s}_{i}(-\tilde{\partial}) \tau_{N-1}(t) z^{N-1-i} \\
& =b_{0} \ldots b_{\frac{N-3}{2}} \sum_{i=0}^{N-1} \mathbf{s}_{i}(-\tilde{\partial}) \mathbf{s}_{\left(1^{\frac{N-1}{2}}\right)}(t) z^{N-1-i} \\
& =b_{0} \ldots b_{\frac{N-3}{2}} \sum_{i=0}^{\frac{N-1}{2}} z^{N-1-i}(-1)^{i} \mathbf{s}_{\left(1^{\frac{N-1}{2}-i}\right)}(t)
\end{aligned}
$$

using Lemma 4.1, and so

$$
\tilde{Q}_{N, N-i}=(-1)^{i}\left(\prod_{0}^{\frac{N-3}{2}} b_{k}\right) \mathbf{s}_{\left(1^{\frac{N-1}{2}-i}\right)} .
$$

So, the last row of $\tilde{Q}$ reads

$$
\prod_{0}^{\frac{N-3}{2}} b_{i}(\underbrace{0, \ldots, 0}_{\frac{N-1}{2}},(-1)^{\frac{N-1}{2}},(-1)^{\frac{N-3}{2}} \mathbf{s}_{1}(t),(-1)^{\frac{N-5}{2}} \mathbf{s}_{\left(1^{2}\right)}(t), \ldots, \mathbf{s}_{\left(1^{\frac{N-1}{2}}\right)}(t))
$$

and and the last row of $Q=D \tilde{Q}$ :

$$
\begin{aligned}
& Q_{N, N-i}=(D \tilde{Q})_{N, N-i}=(-1)^{i} \prod_{0}^{\frac{N-3}{2}} b_{k} \frac{\mathbf{s}\left(1^{\frac{N-1}{2}-i}\right)}{\sqrt{\tau_{N-1}}} \\
&=(-1)^{i}\left(\prod_{0}^{N-3} b_{k}\right)^{1 / 2} \frac{\mathbf{s}\left(1^{\frac{N-1}{2}-i}\right)}{(t)} \\
&\left(\mathbf{s}_{\left(1^{\frac{N-1}{2}}\right)}(t)\right)^{1 / 2}
\end{aligned}
$$


and so, using Lemma 4.1,

$$
\frac{\partial Q_{N, N}}{\partial t_{i}}=-\frac{(-1)^{i}}{2}\left(\prod_{0}^{N-3} b_{k}\right)^{1 / 2} \frac{\mathbf{s}_{\left(1^{\frac{N-1}{2}-i}\right)}(t)}{\left(\mathbf{s}_{\left(1^{\frac{N-1}{2}}\right)}(t)\right)^{1 / 2}}=-\frac{1}{2} Q_{N, N-i} .
$$

Having checked (2.0.6), (2.0.7) and (2.0.8) (in the odd case) of Theorem 2.2, we have found a solution of the Pfaff lattice. This finally concludes the proof of Theorem 1.1.

Proof of Theorem 1.2: According to [2], Pfaff $\tau$-functions satisfy bilinear relations: for all $t, t^{\prime} \in \mathbb{C}^{\infty}$ and $m, n$ positive integers

$$
\begin{aligned}
\oint_{z=\infty} \tau_{2 n}(t- & {\left.\left[z^{-1}\right]\right) \tau_{2 m+2}\left(t^{\prime}+\left[z^{-1}\right]\right) e^{\sum_{i=0}^{\infty}\left(t_{i}-t_{i}^{\prime}\right) z^{i}} z^{2 n-2 m-2} d z } \\
& +\oint_{z=0} \tau_{2 n+2}(t+[z]) \tau_{2 m}\left(t^{\prime}-[z]\right) e^{\sum_{i=0}^{\infty}\left(t_{i}^{\prime}-t_{i}\right) z^{-i}} z^{2 n-2 m} d z=0,
\end{aligned}
$$

Shifting appropriately and taking residues leads to the "differential Fay identity":

$$
\begin{aligned}
& \left\{\tau_{2 n}(t-[u]), \tau_{2 n}(t-[v])\right\} \\
& \quad+\left(u^{-1}-v^{-1}\right)\left(\tau_{2 n}(t-[u]) \tau_{2 n}(t-[v])-\tau_{2 n}(t) \tau_{2 n}(t-[u]-[v])\right) \\
& \quad=u v(u-v) \tau_{2 n-2}(t-[u]-[v]) \tau_{2 n+2}(t)
\end{aligned}
$$

and Hirota bilinear equations, involving nearest neighbors:

$$
\left(\mathbf{s}_{k+4}(\tilde{\partial})-\frac{1}{2} \frac{\partial}{\partial t_{1}} \frac{\partial}{\partial t_{k+3}}\right) \tau_{2 n} \cdot \tau_{2 n}=\mathbf{s}_{k}(\tilde{\partial}) \tau_{2 n+2} \cdot \tau_{2 n-2} .
$$

It only remains to check the "boundary condition":

$$
\begin{cases}\tau_{N}=\prod_{0}^{\frac{N-2}{2}} b_{i}, & \text { for even } N \\ \tau_{N+1}=0, & \text { for odd } N\end{cases}
$$

\footnotetext{
${ }^{10} \tilde{\partial}=\left(\partial / \partial t_{1},(1 / 2) \partial / \partial t_{2},(1 / 3) \partial / \partial t_{3}, \ldots\right), \tilde{D}=\left(D_{1},(1 / 2) D_{2},(1 / 3) D_{3}, \ldots\right)$ is the corresponding Hirota symbol: $P(\tilde{D}) f \cdot g:=\left.P\left(\partial / \partial y_{1},(1 / 2) \partial / \partial y_{2}, \ldots\right) f(t+y) g(t-y)\right|_{y=0}$, and $\mathbf{s}_{k}$ are the previously defined elementary Schur functions: $\sum_{k=0}^{\infty} \mathbf{s}_{k}(t) z^{k}:=\exp \left(\sum_{i=1}^{\infty} t_{i} z^{i}\right)$. For further notations, see Dickey $[5]$.
} 
Indeed, for even $N$, using $\operatorname{det} E_{N N}(t)=1$ and the matrix (3.0.2), we have that

$$
\left(p f m_{N}(t)\right)^{2}=\operatorname{det}\left(E_{N, N}(t) m_{N}(0) E_{N, N}^{\top}(t)\right)=\operatorname{det} m_{N}(0)=\prod_{0}^{\frac{N-2}{2}} b_{i} .
$$

Moreover, for odd $N$, according to (4.0.4), $\tau_{N-1}$ is a pure Schur polynomial, which is known to satisfy the KP Fay identity; i.e., the equation (4.0.5), without right hand side. This justifies setting $\tau_{N+1}=0$ for odd $N$.

In the next Proposition, we show that the finite vector of skew-orthogonal polynomials form an eigenvector of the matrix $L$, with modified boundary condition.

Proposition 4.2 For even $N$, the skew-orthonormal polynomials $q=\left(q_{0}\right.$, . ..,$\left.q_{N-1}\right)^{\top}=Q \quad\left(1, \ldots, z^{N-1}\right)^{\top}$ are eigenfunctions for $L$, with the boundary condition:

$$
L q=z q-\left(0, \ldots, 0, z^{N}\right) \sqrt{p f m_{N-2}}\left(\prod_{0}^{\frac{N-2}{2}} b_{i}\right)^{-1 / 2}
$$

Proof: Indeed

$$
\begin{aligned}
L q & =Q \Lambda Q^{-1} Q\left(\begin{array}{c}
1 \\
\vdots \\
z^{N-1}
\end{array}\right) \\
& =Q \Lambda\left(\begin{array}{c}
1 \\
\vdots \\
z^{N-1}
\end{array}\right) \\
& =Q z\left(\begin{array}{c}
1 \\
\vdots \\
z^{N-2} \\
0 \\
q_{0} \\
q_{1} \\
\vdots \\
q_{N-2} \\
\bar{q}_{N-1}
\end{array}\right)=z q+z\left(0, \ldots, 0, \bar{q}_{N-1}-q_{N-1}\right) \\
& =z\left(\begin{array}{c} 
\\
q^{N}
\end{array}\right)
\end{aligned}
$$


where $\bar{q}_{N-1}$ is the same as $q_{N-1}$, but without leading term, i.e., $\bar{q}_{N-1}=$ $q_{N-1}-Q_{N N} z^{N-1}$, where by (4.0.7) we have

$$
Q_{N N}=\sqrt{\frac{\tau_{N-2}}{\tau_{N}}}=\sqrt{p f m_{N-2}}\left(\prod_{0}^{\frac{N-2}{2}} b_{i}\right)^{-1 / 2}
$$

ending the proof of Proposition 4.2.

\section{$5 \quad$ Vertex operators}

The purpose of this section is to prove Theorem 1.3 and Corollary 1.4. Define as in (1.0.23),

$$
\beta:=\frac{N}{2}-\ell+1
$$

Remembering from (1.0.21) the vertex operator $X(t ; z)$, consider now its formal expansion in powers of $z$

$$
X(t ; z)=e^{\sum_{1}^{\infty} t_{i} z^{i}} e^{-\sum_{1}^{\infty} \frac{z^{-i}}{i} \frac{\partial}{\partial t_{i}}}=: \sum_{i \in \mathbb{Z}} B_{i} z^{i},
$$

with differential operators (see footnote 10)

$$
B_{i}:=\left.B_{i}^{(\alpha)}\right|_{\alpha=1} \text { and } B_{i}^{(\alpha)}:=\sum_{j \geq 0} \mathbf{s}_{i+j}(\alpha t) \mathbf{s}_{j}\left(-\alpha \tilde{\partial}_{t}\right) .
$$

Also define as in (1.0.22) the vector vertex operator $\square$

$$
\mathbb{X}(t ; z)=\Lambda^{\top} e^{\sum_{1}^{\infty} t_{i} z^{i}} e^{-\sum_{1}^{\infty} \frac{z^{-i}}{i} \frac{\partial}{\partial t_{i}}} \chi(z) .
$$

Also remember the definitions of the integrated vertex operator, in terms of the vertex operator (5.0.2) and a function $\rho_{b}$, defined in (5.0.8) below,

$$
Y_{\beta}(t):=\frac{1}{(2 \pi i)^{2}} \oint_{\infty} \oint_{\infty} X(t ; y) X(t ; z) \frac{\rho_{b}(y / z) d y d z}{z^{2}(y z)^{\beta}}
$$

\footnotetext{
${ }^{11} \chi(z):=\left(z^{i}\right)_{i \geq 0}$
} 
and the integrated vector vertex operator, in terms of (5.0.4),

$$
\mathbb{Y}_{N}(t)=\frac{1}{(2 \pi i)^{2}} \oint_{\infty} \oint_{\infty} \mathbb{X}(t ; y) \mathbb{X}(t ; z) \frac{\rho_{b}(y / z) d y d z}{2(y z)^{N / 2} z} .
$$

In both cases, the double integral around two contours about $\infty$ amounts to computing the coefficient of $1 / y z$. The next Theorem is nothing but a rephrasing of Theorem 1.3 and Corollary 1.4.

Theorem 5.1 For a given set of $b_{i}$, the sequence of $\tau$-functions $\tau_{0}, \tau_{2}, \tau_{4}, \ldots$, defined in 1.0.15), is generated by the vertex operators $Y_{\beta}$ :

$$
Y_{\beta} \tau_{\ell-2}=\ell \tau_{\ell}
$$

The vector $I=\left(I_{0}, I_{2}, I_{4}, \ldots\right)$, with $I_{\ell}=\left(\frac{\ell}{2}\right) ! \tau_{\ell}$ is a fixed point for the vector vertex operator $\mathbb{Y}_{N}$, namely

$$
\left(\mathbb{Y}_{N} I\right)_{\ell}=I_{\ell}, \text { for even } \ell
$$

We shall first need a few propositions.

Proposition 5.2 Defining

$$
\rho_{b}(x):=\left\{\begin{array}{l}
\rho_{b}^{(e)}(x):=\sum_{i \geq 0} b_{i}\left(x^{-i-1}-x^{i}\right), \text { for } N \text { even }, \\
\rho_{b}^{(o)}(x):=x^{-1 / 2} \sum_{i \geq 0} b_{i}\left(x^{-i-1}-x^{i+1}\right), \text { for } N \text { odd },
\end{array}\right.
$$

we have

$$
\begin{aligned}
Y_{\beta}(t)= & \frac{1}{(2 \pi i)^{2}} \oint_{\infty} \oint_{\infty} X(t ; y) X(t ; z) \frac{\rho_{b}(y / z) d y d z}{z^{2}(y z)^{\beta}} \\
= & \begin{cases}\sum_{j \geq 0} b_{j}\left(B_{\beta+j} B_{\beta-j}-B_{\beta-j-1} B_{\beta+j+1}\right), \text { for } N \text { even } \\
\sum_{j \geq 0} b_{j}\left(B_{\beta+j+1 / 2} B_{\beta-j-1 / 2}-B_{\beta-j-3 / 2} B_{\beta+j+3 / 2}\right), \text { for } N \text { odd }\end{cases}
\end{aligned}
$$


Proof: Compute for $N$ even,

$$
\begin{aligned}
\frac{X(t ; y) X(t ; z)}{(y z)^{\beta}} & =\sum_{i \in \mathbb{Z}} B_{i} y^{i-\beta} \sum_{j \in \mathbb{Z}} B_{j} z^{j-\beta} \\
& =\sum_{i \in \mathbb{Z}} B_{\beta+i} y^{i} \cdot \sum_{j \in \mathbb{Z}} B_{\beta-j} z^{-j} \\
& =\sum_{i, j \in \mathbb{Z}} B_{\beta+i} B_{\beta-j} \frac{y^{i}}{z^{j}} \\
& =\sum_{j \in \mathbb{Z}} B_{\beta+j} B_{\beta-j}\left(\frac{y}{z}\right)^{j}+\sum_{i \neq j \in \mathbb{Z}} a_{i j} \frac{y^{i}}{z^{j}}
\end{aligned}
$$

and so,

$$
\begin{aligned}
& \rho_{b}^{(e)}\left(\frac{y}{z}\right) \cdot \frac{X(t ; y) X(t ; z)}{z^{2}(y z)^{\beta}} \\
& =\frac{1}{z^{2}}\left(\sum_{i \geq 0} b_{i}\left[\left(\frac{y}{z}\right)^{-(i+1)}-\left(\frac{y}{z}\right)^{i}\right]\right) \cdot\left(\sum_{j \in \mathbb{Z}} B_{\beta+j} B_{\beta-j}\left(\frac{y}{z}\right)^{j}+\sum_{i \neq j \in \mathbb{Z}} a_{i j} \frac{y^{i}}{z^{j}}\right) \\
& =\frac{1}{y z}\left(\sum_{j \geq 0} b_{j}\left(B_{\beta+j} B_{\beta-j}-B_{\beta-j-1} B_{\beta+j+1}\right)\right)+\sum_{i \text { or } j \neq 0} c_{i j} y^{i-1} z^{j-1}
\end{aligned}
$$

and therefore, upon taking the double residue,

$$
\oint_{\infty} \oint_{\infty} \frac{\rho_{b}^{(e)}(y / z) X(t ; y) X(t ; z)}{z^{2}(y z)^{\beta}} \frac{d y d z}{(2 \pi i)^{2}}=\sum_{j \geq 0} b_{j}\left(B_{\beta+j} B_{\beta-j}-B_{\beta-j-1} B_{\beta+j+1}\right) .
$$

$\underline{\text { For } N \text { odd, }}$

$$
\frac{X(t ; y) X(t ; z)}{(y z)^{\beta}(y / z)^{1 / 2}}=\sum_{j \in \mathbb{Z}} B_{\beta+\frac{1}{2}+j} B_{\beta-\frac{1}{2}-j}\left(\frac{y}{z}\right)^{j}+\sum_{i \neq j \in \mathbb{Z}} a_{i j} \frac{y^{i}}{z^{j}}
$$

and so

$$
\begin{aligned}
& \rho_{b}^{(o)}\left(\frac{y}{z}\right) \frac{X(t ; y) X(t ; z)}{z^{2}(y z)^{\beta}} \\
& \quad=\frac{1}{y z} \sum_{j \geq 0} b_{j}\left(B_{\beta+j+\frac{1}{2}} B_{\beta-j-\frac{1}{2}}-B_{\beta-j-\frac{3}{2}} B_{\beta+j+\frac{3}{2}}\right)+\sum_{i \text { or } j \neq 0} c_{i j} y^{i-1} z^{j-1}
\end{aligned}
$$


and therefore

$\oint_{\infty} \oint_{\infty} \frac{\rho_{b}^{(o)}(y / z) X(t ; y) X(t ; z)}{z^{2}(y z)^{\beta}} \frac{d y d z}{(2 \pi i)^{2}}=\sum_{j \geq 0} b_{j}\left(B_{\beta+j+\frac{1}{2}} B_{\beta-j-\frac{1}{2}}-B_{\beta-j-\frac{3}{2}} B_{\beta+j+\frac{3}{2}}\right)$,

ending the proof of Proposition 5.2.

Defining the set

$$
\mathbb{S}_{N}^{(\ell)}:=\left\{\begin{array}{c}
\sigma_{1}>\sigma_{2}>\ldots>\sigma_{\ell / 2}, \quad \sigma_{i} \in \mathbb{Z} \\
\frac{\ell}{2} \leq \sigma_{i}+i \leq\left[\frac{N}{2}\right]
\end{array}\right\}
$$

the map

$$
\sigma: \mathbb{Y}_{\frac{\ell(N-\ell)}{2}}^{(\ell)} \longrightarrow \mathbb{S}_{N}^{(\ell)}: \lambda \longmapsto \sigma(\lambda)=\left(\lambda_{i}-i+\ell-\left[\frac{N+1}{2}\right]\right)_{1 \leq i \leq n / 2}
$$

is a bijection.

Indeed, $\lambda_{1} \geq \lambda_{2} \geq \ldots$ implies at once the strict inequalities $\sigma_{1}>\sigma_{2}>\ldots$ and also implies, together with the fact that for $\lambda \in \mathbb{Y}_{\frac{\ell(N-\ell)}{2}}^{(\ell)}$ and $1 \leq i \leq \ell / 2$,

$$
2 \lambda_{i} \geq \lambda_{i}+\lambda_{\ell+1-i}=N-\ell \text { and, clearly } \lambda_{i} \leq N-\ell .
$$

Conversely, every $\sigma \in \mathbb{S}_{N}^{(\ell)}$ comes from a $\lambda \in \mathbb{Y}_{\frac{\ell(N-\ell)}{2}}^{(\ell)}$.

Lemma 5.3 For a given partition

$$
\lambda=\left(\lambda_{1} \geq \lambda_{2} \geq \ldots \geq \lambda_{\ell-2}\right) \in \mathbb{Y}_{\frac{(\ell-2)(N-\ell+2)}{2}}^{(\ell-2)},
$$

and $j \geq 0$, the following holds

$$
B_{\beta+j} B_{\beta-j} \mathbf{s}_{\lambda}=-B_{\beta-j-1} B_{\beta+j+1} \mathbf{s}_{\lambda}=\left\{\begin{array}{l}
0, \quad \text { if } \beta+j=\text { some } \lambda_{\nu}-\nu-1 \\
\text { for } 1 \leq \nu \leq \ell / 2-1, \\
\text { or if } j \geq N / 2 \\
\mathbf{s}_{\lambda^{\prime}}, \quad \text { if } \beta+j \neq \text { every } \lambda_{\nu}-\nu-1 \\
\text { for } 1 \leq \nu \leq \ell / 2-1,
\end{array}\right.
$$


where

$$
\begin{aligned}
\lambda^{\prime}=\left(\lambda_{1}-2\right. & \geq \ldots \geq \lambda_{\nu}-2 \geq \beta+j+\nu \geq \lambda_{\nu+1}-1 \geq \ldots \geq \lambda_{\frac{\ell}{2}-1}-1 \\
& \geq \lambda_{\frac{\ell}{2}}-1 \geq \ldots \geq \lambda_{\ell-2-\nu}-1 \geq(N-\ell)-(\beta+j+\nu) \\
& \left.\geq \lambda_{\ell-1-\nu} \geq \ldots \geq \lambda_{\ell-2}\right) \in \mathbb{Y}_{\frac{\ell(N-\ell)}{2}}^{(\ell)}
\end{aligned}
$$

Moreover for $j$ 's such that $\beta+j \neq$ every $\lambda_{\nu}-\nu-1$, the maps $B_{\beta+j} B_{\beta-j}$ induce maps

$$
B_{\beta+j} B_{\beta-j}: \quad \mathbb{Y}_{\frac{(\ell-2)(N-\ell+2)}{2}}^{(\ell-2)} \longrightarrow \mathbb{Y}_{\frac{\ell(N-\ell)}{2}}^{(\ell)}: \lambda \longmapsto \lambda^{\prime}
$$

having, as a whole, a "surjectivity property", meaning that to each $\lambda^{\prime} \in$ $\mathbb{Y}_{(\ell-2)(N-\ell) / 2}^{\ell}$, there are $\ell \overline{/ 2 \text { choices of } j \geq 0}$ and $\lambda \in \mathbb{Y}_{(\ell-2)(N-\ell+2) / 2}^{(\ell-2)}$ mapping to $\lambda^{\prime}$, by means of the map $B_{\beta+j} B_{\beta-j}$, as in (5.0.12).

At the level of the $\mathbb{S}$-spaces, the maps $B_{\beta+j} B_{\beta-j}$ induce maps

$$
\mathbb{S}_{N}^{(\ell-2)} \longrightarrow \mathbb{S}_{N}^{(\ell)}: \sigma=\left(\sigma_{1}, \ldots, \sigma_{\frac{\ell-2}{2}}\right) \longmapsto \sigma^{\prime}=\left(\sigma_{1}, \ldots, \sigma_{\nu}, j, \sigma_{\nu+1}, \ldots, \sigma_{\frac{\ell-2}{2}}\right),
$$

having the same "surjectivity property" as above.

For $N$ odd, all formulae above remain the same, except for the substitution $j \mapsto j+\frac{1}{2}$ in (5.0.19) and (5.0.13).

Proof: Extending a classic identity (see MacDonald \&) to arbitrary sequences $\left(\lambda_{1}, \ldots, \lambda_{n}\right)$, we have

$$
B_{\lambda_{1}}, \ldots, B_{\lambda_{n}}(1)=\left(\lambda_{1}, \ldots, \lambda_{n}\right):=\operatorname{det}\left(\mathbf{s}_{\lambda_{i}+j-i}(t)\right)_{1 \leq i, j \leq n}
$$

and, in particular, for a partition $\left(\lambda_{1} \geq \lambda_{2} \geq \ldots \geq \lambda_{\ell}\right)$, we have, for an arbitrary choice of $j \geq 0$,

$$
\begin{aligned}
& B_{\beta+j} B_{\beta-j} \mathbf{s}_{\left(\lambda_{1}, \ldots, \lambda_{\ell-2}\right)} \mathbf{s}_{\left(\beta+j, \beta-j, \lambda_{1}, \ldots, \lambda_{\ell-2}\right)} \\
&=\operatorname{det}\left(\begin{array}{ccccc}
\mathbf{s}_{\beta+j} & \mathbf{s}_{\beta+j+1} & \mathbf{s}_{\beta+j+2} & \ldots & \mathbf{s}_{\beta+j+\ell-1} \\
\mathbf{s}_{\beta-j-1} & \mathbf{s}_{\beta-j} & \mathbf{s}_{\beta-j+1} & \ldots & \mathbf{s}_{\beta-j+\ell-2} \\
\mathbf{s}_{\lambda_{1}-2} & \mathbf{s}_{\lambda_{1}-1} & \mathbf{s}_{\lambda_{1}} & \ldots & \mathbf{s}_{\lambda_{1}+\ell-3} \\
\vdots & & & \ddots & \vdots \\
\mathbf{s}_{\lambda_{\ell-2}-\ell+1} & \ldots & \ldots & \ldots & \mathbf{s}_{\lambda_{\ell-2}}
\end{array}\right) .
\end{aligned}
$$


Using the value (5.0.1) of $\beta$, it is immediately clear, from the matrix (5.0.16), that for $j \geq N / 2$, the second row of the matrix (5.0.16) vanishes and therefore the determinant. Therefore we assume $0 \leq j \leq \frac{N}{2}-1$. We give the proof for even $N$; for odd $N$, it is identical with $j \mapsto j+1 / 2$.

The first column of the matrix above involves the indices

$$
\frac{N}{2}-\ell+1+j, \frac{N}{2}-\ell-j, \lambda_{1}-2, \lambda_{2}-3, \ldots, \lambda_{\frac{\ell}{2}}-\frac{\ell}{2}-1, \ldots, \lambda_{\ell-2}-\ell+1 .
$$

Consider now an arbitrary integer $j \geq 0$ and an arbitrary partition

$$
\lambda \in \mathbb{Y}_{\frac{(\ell-2)(N-\ell+2)}{2}}^{(\ell-2)}
$$

it has the property that

$$
\lambda_{i}+\lambda_{\ell-1-i}=N-\ell+2 \text { for } 1 \leq i \leq \frac{\ell-2}{2} .
$$

Hence, for $i=\frac{\ell-2}{2}$

$$
2 \lambda_{\frac{\ell}{2}} \leq \lambda_{\frac{\ell}{2}-1}+\lambda_{\frac{\ell}{2}}=N-\ell+2
$$

and so

$$
\lambda_{\ell / 2} \leq \frac{N-\ell+2}{2}
$$

thus, for the arbitrary $j \geq 0$ chosen above

$$
\lambda_{\ell / 2}-\ell / 2-1 \leq \frac{N}{2}-\ell<\frac{N}{2}-\ell+j+1 .
$$

The partition $\lambda_{1} \geq \lambda_{2} \geq \ldots$ implies the strict inequalities

$\lambda_{1}-1-1>\lambda_{2}-2-1>\lambda_{3}-3-1>\ldots>\lambda_{\nu+1}-(\nu+1)-1>\ldots>\lambda_{\ell / 2}-\ell / 2-1$

and therefore, there exist $0 \leq \nu \leq \frac{\ell}{2}-1$ such that

$$
\lambda_{\nu}-\nu-1 \geq \frac{N}{2}-\ell+j+1 \geq \lambda_{\nu+1}-\nu-2 .
$$

These inequalities together with the fact that

$$
\lambda_{\nu}+\lambda_{\ell-1-\nu}=N-\ell+2, \quad \lambda_{\nu+1}+\lambda_{\ell-2-\nu}=N-\ell+2
$$


also imply

$$
\lambda_{\ell-2-\nu}-(\ell-1-\nu) \geq \frac{N}{2}-\ell-j \geq \lambda_{\ell-1-\nu}-(\ell-\nu) .
$$

Therefore, the indices (5.0.17) of the first column of the matrix (5.0.16) are now rearranged by order, as follows:

$$
\begin{aligned}
& \lambda_{1}-2>\lambda_{2}-3>\ldots>\lambda_{\nu}-\nu-1 \geq \frac{N}{2}-\ell+1+j \geq \lambda_{\nu+1}-\nu-2 \\
& >\ldots>\lambda_{\frac{\ell}{2}-1}-\frac{\ell}{2}>\lambda_{\frac{\ell}{2}}-\frac{\ell}{2}-1>\ldots>\lambda_{\ell-2-\nu}-(\ell-1-\nu) \\
& \geq \frac{N}{2}-\ell-j \geq \lambda_{\ell-1-\nu}-(\ell-\nu)>\ldots>\lambda_{\ell-2}-\ell+1
\end{aligned}
$$

Notice that the determinant (5.0.16) vanishes if any of the equalities hold in (5.0.18) above. Therefore we may assume strict inequalities. Upon rearranging the rows of the matrix (5.0.16) according to the order in (5.0.18), we now list the corresponding partitions by looking at the indices on the diagonal. This amounts to adding $i-1$ to the $i^{\text {th }}$ entry of (5.0.18), thus leading to

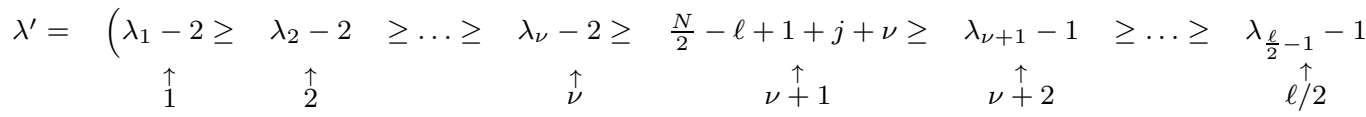

$$
\begin{aligned}
& \left.\geq \lambda_{\frac{\ell}{2}}-1 \quad \geq \ldots \geq \quad \lambda_{\ell-2-\nu}-1 \geq \quad \frac{N}{2}-j-\nu-1 \geq \quad \lambda_{\ell-1-\nu} \quad \geq \ldots \geq \lambda_{\ell-2}\right)
\end{aligned}
$$

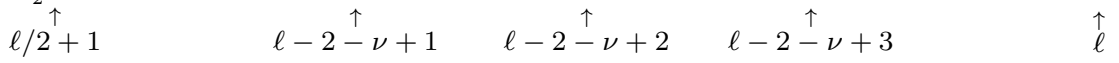

The rearrangement does not change the sign of the determinant (5.0.16). Knowing that $\lambda \in \mathbb{Y}_{\frac{(\ell-2)(N-\ell+2)}{2}}^{(\ell-2)}$, we now prove that the new partition $\lambda^{\prime}$ (obtained in (5.0.19) ),

$$
\lambda^{\prime} \in \mathbb{Y}_{\frac{\ell(N-\ell)}{2}}^{(\ell)}
$$


i.e., we prove

$$
\begin{aligned}
\sum_{i}^{\ell} \lambda_{i}^{\prime} & =\sum_{1}^{\ell-2} \lambda_{i}-2 \nu-(\ell-2-2 \nu)+\left(\frac{N}{2}-\ell+1+j+\nu\right) \\
& +\left(\frac{N}{2}-j-\nu-1\right) \\
& =\frac{\ell(N-\ell)}{2}
\end{aligned}
$$

and

$$
\lambda_{i}^{\prime}+\lambda_{\ell+1-i}^{\prime}=N-\ell \quad \text { for all } 1 \leq i \leq \frac{\ell}{2}
$$

e.g.,

$$
\begin{aligned}
\lambda_{i}^{\prime}+\lambda_{\ell+1-i}^{\prime} & =\lambda_{i}-2+\lambda_{\ell-1-i}=N-\ell \text { for } 1 \leq i \leq \nu \\
\lambda_{\nu+1}^{\prime}+\lambda_{\ell-\nu}^{\prime} & =\left(\frac{N}{2}-\ell+1+j+\nu\right)+\left(\frac{N}{2}-j-\nu-1\right)=N-\ell \\
\lambda_{i}^{\prime}+\lambda_{\ell+1-i}^{\prime} & =\lambda_{i-1}-1+\lambda_{\ell-i}-1=N-\ell \text { for } \nu+2 \leq i \leq \ell / 2 .
\end{aligned}
$$

So far, we have shown that to an arbitrary integer $j \geq 0$ and a partition

$$
\lambda=\left(\lambda_{1} \geq \lambda_{2} \geq \ldots \geq \lambda_{\ell-2}\right) \in \mathbb{Y}_{\frac{(\ell-2)(N-\ell+2)}{2}}^{(\ell-2)},
$$

such that the inequalities in $(5.0 .19)$ are strict, corresponds a new partition

$$
\lambda^{\prime}=\left(\lambda_{1}^{\prime} \geq \ldots \geq \lambda_{\ell}^{\prime}\right) \in \mathbb{Y}_{\frac{\ell(N-\ell)}{2}}^{(\ell)},
$$

with $\lambda^{\prime}$ totally determined by (5.0.19). Then $\ell / 2$ different choices of $\lambda \in$ $\mathbb{Y}_{(\ell-2)(N-\ell+2) / 2}^{(\ell-2)}$ and $j \geq 0$ will lead to the same sequence of numbers (5.0.19), as appears from the next argument.

In view of the $\sigma$-map in (5.0.11), it is obvious to see that the $\nu+1$-st number in $\lambda^{\prime}$ of (5.0.19) gets mapped by $\sigma$ into $j$, namely

$$
\frac{N}{2}-\ell+1+j+\nu \longmapsto j
$$

and, in general, (5.0.15) holds. The "surjectivity property" is straightforward in this description, since given a sequence $\sigma^{\prime} \in \mathbb{S}_{N}^{(\ell)}$, you may choose $j$ to be any of the $\ell / 2$ numbers appearing in $\sigma^{\prime}$; then $\sigma$ is the sequence formed by the remaining numbers in order. This establishes Lemma 5.3. 
Proposition 5.4 Given positive integers $N$ and $\ell$ with even $\ell$ and the operator

$$
Y_{\beta}=\left\{\begin{array}{l}
\sum_{j \geq 0} b_{j}\left(B_{\beta+j} B_{\beta-j}-B_{\beta-j-1} B_{\beta+j+1}\right), \quad N \text { even }, \\
\sum_{j \geq 0} b_{j}\left(B_{\beta+j+\frac{1}{2}} B_{\beta-j-\frac{1}{2}}-B_{\beta-j-\frac{3}{2}} B_{\beta+j+\frac{3}{2}}\right), \quad N \text { odd },
\end{array}\right.
$$

we have

$$
Y_{\frac{N}{2}-\ell+1} \tau_{\ell-2}=\ell \tau_{\ell}
$$

Proof: The indices of the $b_{i}$ in the $\ell$ th tau-function can now be expressed in terms of the $\sigma$-map, as follows

$$
\tau_{\ell}(t)=\sum_{\lambda \in \mathbb{Y}_{\frac{\ell(N-\ell)}{2}}^{(\ell)}}\left(\prod_{1}^{\ell / 2} b_{\lambda_{i}-i+\ell-\left[\frac{N+1}{2}\right]}\right) \mathbf{s}_{\lambda}(t)=\sum_{\lambda \in \mathbb{Y}_{\frac{\ell(N-\ell)}{2}}^{(\ell)}}\left(\prod_{1}^{\ell / 2} b_{\sigma_{i}(\lambda)}\right) \mathbf{s}_{\lambda}(t) .
$$

We give the proof for even $N$. From (5.0.15), it follows at once that

$$
b_{j} \prod_{1}^{(\ell-2) / 2} b_{\sigma_{i}(\lambda)}=\prod_{1}^{\ell / 2} b_{\sigma_{i}\left(\lambda^{\prime}\right)} .
$$

Setting $Y_{\beta}=\sum_{i \geq 0} b_{i} \Gamma_{i}$, one computes, using Lemma 5.3, (5.0.20) and in $\stackrel{*}{=}$ the $\ell / 2$-to-1 "surjectivity" of the maps (5.0.14) or (5.0.15):

$$
\begin{aligned}
Y_{\frac{N}{2}-\ell+1} \tau_{\ell-2}(t) & =\sum_{\lambda \in \mathbb{Y}_{\frac{(\ell-2)(N-\ell-2)}{2}}^{(\ell-2)}}\left(\prod_{1}^{\frac{\ell-2}{2}} b_{\sigma_{i}(\lambda)}\right) Y_{\beta}\left(s_{\lambda}(t)\right) \\
& =\sum_{\lambda \in \mathbb{Y}_{\frac{(\ell-2)(N-\ell-2)}{2}}^{(\ell-2)}} \sum_{j \geq 0}\left(\prod_{1}^{\frac{\ell-2}{2}} b_{\sigma_{i}(\lambda)}\right) b_{j} \Gamma_{j}\left(s_{\lambda}(t)\right) \\
& \stackrel{*}{=} \sum_{\lambda^{\prime} \in \mathbb{Y}_{\frac{\ell(N-\ell)}{2}}^{\ell}} \prod_{1}^{\ell / 2} b_{\sigma_{i}\left(\lambda^{\prime}\right)} 2 s_{\lambda^{\prime}}(t) \\
& =\ell \tau_{\ell}(t),
\end{aligned}
$$


ending the proof of Proposition 5.4.

Proof of Theorem 5.1: Formula (5.0.6) follows at once from Propositions 5.2 and 5.4. To prove (5.0.7), first notice that, upon setting $I_{\ell}:=\left(\frac{\ell}{2}\right) ! \tau_{\ell}$,

$$
(\mathbb{X}(t ; y) \mathbb{X}(t ; z) I)_{\ell}=y^{\ell-1} z^{\ell-2} X(t ; y) X(t ; z) I_{\ell-2}
$$

Then

$$
\begin{aligned}
(\mathbb{Y}(t) I)_{\ell} & =\left(\frac{1}{(2 \pi i)^{2}} \oint_{\infty} \oint_{\infty} \mathbb{X}(t ; y) \mathbb{X}(t ; z) \frac{\rho_{b}(y / z) d y d z}{2 z(y z)^{N / 2}} I\right)_{\ell} \\
& =\frac{1}{(2 \pi i)^{2}} \oint_{\infty} \oint_{\infty} \frac{d y d z \rho_{b}(y / z)}{2 z^{2}(y z)^{N / 2-\ell+1} X(t ; y) X(t ; z) I_{\ell-2}} \\
& =\frac{1}{2} Y_{\frac{N}{2}-\ell+1} I_{\ell-2}, \text { by definition }(5.0 .5) \text { of } Y_{\beta}, \\
& =\frac{1}{2} Y_{\frac{N}{2}-\ell+1}\left(\frac{\ell-2}{2}\right) ! \tau_{\ell-2} \\
& =\left(\frac{\ell}{2}\right) ! \tau_{\ell}, \quad \text { using }(5.0 .6) \\
& =I_{\ell},
\end{aligned}
$$

ending the proof of Theorem 5.1.

Example: For $b_{i}=2 i+1$ and even $N$, the function $\rho_{b}(x)$, defined in (5.0.8), equalst2

$$
\rho_{b}(x)=\sum_{i \geq 0} b_{i}\left(x^{-i-1}-x^{i}\right)=-\frac{1+x}{(1-x)^{2}}+x^{-1} \frac{1+x^{-1}}{\left(1-x^{-1}\right)^{2}} .
$$

The corresponding vertex operator (5.0.9) takes on a particularly simple form:

$$
Y_{\frac{N}{2}-\ell+1}=2 B_{N-2 \ell+2}^{(2)}=2 \int_{\mathbb{R}} d u \delta^{(N-2)}(u) u^{2 \ell-4} X^{(2)}(u),
$$

\footnotetext{
${ }^{12} \rho_{b}(x)$ is actually a distribution!
} 
where $\delta^{(N-2)}$ is the $(N-2) n d$ derivative of the customary $\delta$-function and where the $B_{i}^{(2)}$ are the differential operators (5.0.3) in the $t_{i}$,

$$
B_{i}^{(2)}:=\sum_{j \geq 0} \mathbf{s}_{i+j}(2 t) \mathbf{s}_{j}\left(-2 \tilde{\partial}_{t}\right)
$$

given by the coefficients of the expansion in powers of $z$ of the vertex operator

$$
X^{(2)}(z):=e^{2 \sum_{1}^{\infty} t_{i} z^{i}} e^{-2 \sum_{1}^{\infty} \frac{z^{-i}}{i} \frac{\partial}{\partial t_{i}}}=\sum_{i \in \mathbb{Z}} B_{i}^{(2)} z^{i} .
$$

Proof: Formula (5.0.21) follows immediately from the series

$$
\frac{1+x}{(1-x)^{2}}=1+3 x+5 x^{2}+7 x^{3}+\ldots .
$$

Setting, for convenience,

$$
X(t ; y, z):=e^{\sum_{1}^{\infty} t_{i}\left(y^{i}+z^{i}\right)} e^{-\sum_{1}^{\infty}\left(\frac{y^{-i}+z^{-i}}{i}\right) \frac{\partial}{\partial t_{i}}}
$$

and using $X(t ; y) X(t ; z)=\left(1-\frac{z}{y}\right) X(t ; y, z)$ and $X(t ; z, z)=X^{(2)}(t ; z)$, one 
computes $\left(\beta=\frac{N}{2}-\ell+1\right)$

$$
\begin{aligned}
Y_{\beta} & =\frac{1}{(2 \pi i)^{2}} \oint_{\infty} \oint_{\infty} \frac{\rho^{e}(y / z)}{(y z)^{\beta} z^{2}} X(t ; y) X(t ; z) d y d z \\
& =\frac{1}{(2 \pi i)^{2}} \oint_{\infty} \oint_{\infty}\left(\frac{z(1+z / y)}{y(1-z / y)^{2}}-\frac{(1+y / z)}{(1-y / z)^{2}}\right) \frac{(1-z / y)}{z^{2}(z y)^{\beta}} X(t ; y, z) d y d z \\
& =\frac{1}{(2 \pi i)^{2}} \oint_{\infty} \oint_{\infty} \frac{z}{y} \frac{(1+z / y)}{(1-z / y)^{2}} \frac{(1-z / y)}{z^{2}(z y)^{\beta}} X(t ; y, z) d y d z \\
& \left.=\oint_{\infty} \oint_{\infty} \frac{(1+z / y)}{(y-z) z(z y)^{\beta}} X(t ; y, z) \frac{d y}{2 \pi i}\right) \frac{d z}{2 \pi i} \\
& =2 \oint_{\infty} \frac{X(t ; z, z)}{z^{2 \beta+1}} \frac{d z}{2 \pi i} \\
& =2 \oint_{\infty} \frac{X^{(2)}(t ; z)}{z^{2 \beta+1}} \frac{d z}{2 \pi i} \\
& =2 B_{2 \beta}^{(2)}=2 B_{N-2 \ell+2}^{(2)}=2 \int_{\mathbb{R}} d u \delta^{(N-2)}(u) u^{2 \ell-4} X^{(2)}(t ; u),
\end{aligned}
$$

establishing (5.0.22).

\section{Duality}

Proposition 6.1 For odd $N$ and odd $\ell$, the following holds:

$$
\begin{aligned}
\tilde{\tau}_{\ell}(t) & :=z^{-1} \operatorname{det}^{1 / 2}\left(E_{\ell, N}(t)\left(m_{N}(0)+z^{2} \varepsilon_{\frac{N+1}{2}, \frac{N+1}{2}}\right) E_{\ell, N}^{\top}(t)\right) \\
& =\sum_{\lambda \in \mathbb{Y}_{\frac{\ell(N-\ell)}{2}}^{(\ell)}}\left(\prod_{1}^{[\ell / 2]} b_{\lambda_{i}-i+\ell-\left[\frac{N+1}{2}\right]}\right) \mathbf{s}_{\lambda_{1} \geq \ldots \geq \lambda_{\ell}}(t) .
\end{aligned}
$$


Then these functions

$$
\tilde{\tau}_{\ell}(t)=(-1)^{\ell(N-\ell) / 2}\left(\prod_{0}^{\frac{N-3}{2}} b_{i}\right)\left(\left.\tau_{N-\ell}(-t)\right|_{b_{i} \rightarrow b_{i}^{-1}}\right), \quad \text { for } \ell \text { odd } .
$$

are the $\tau$-functions $\tau_{k}(t)$ (in reverse order and modulo a multiplicative factor) of the Pfaff lattice for odd $N$ and even $k$, with $t \mapsto-t$, and with initial condition

$$
\left(\begin{array}{cccc}
O & & \\
& & b_{0}^{-1} & \\
& & 0 & \\
-b_{\frac{N-3}{2}}^{-1} & & \\
-b_{\frac{N-3}{2}}^{-1} & & &
\end{array}\right)
$$

Proof: Defining $k_{i}$ and $k_{i}^{\top}$ by

$$
\lambda_{i}=k_{i}-\ell+i, \quad \lambda_{i}^{\top}=k_{i}^{\top}-(N-\ell)+i,
$$

it is easy to see the one-to-one correspondence between

$$
\mathbb{Y}_{\frac{\ell(N-\ell)}{2}}^{(\ell)} \longleftrightarrow\left\{\begin{array}{l}
N-1 \geq k_{1}>k_{2}>\ldots>k_{\ell} \geq 0 \\
\text { with } k_{i}+k_{\ell+1-i}=N-1 \text { for } 1 \leq i \leq \frac{\ell+1}{2}
\end{array}\right\}
$$

and also between

$$
\mathbb{Y}_{\frac{\ell(N-\ell)}{2}}^{(N-\ell)} \longleftrightarrow\left\{\begin{array}{l}
N-1 \geq k_{1}^{\top}>k_{2}^{\top}>\ldots>k_{N-\ell}^{\top} \geq 0 \\
\text { with } k_{i}^{\top}+k_{N-\ell+1-i}^{\top}=N-1 \text { for } 1 \leq i \leq \frac{N-\ell}{2}
\end{array}\right\}
$$

Lemma 6.2 (1) The following correspondence holds

$$
\lambda \in \mathbb{Y}_{\frac{\ell(N-\ell)}{2}}^{(\ell)} \longleftrightarrow \lambda^{\top} \in \mathbb{Y}_{\frac{\ell(N-\ell)}{2}}^{(N-\ell)},
$$

(2) For $\lambda$ and $\lambda^{\top}$, we have the following disjoint union

$$
\left\{k_{1}>\ldots>k_{\ell}\right\} \cup\left\{k_{1}^{\top}>\ldots>k_{N-\ell}^{\top}\right\}=\{0,1, \ldots, N-1\} .
$$




\section{Proof: Considering}

$$
\left(\lambda_{1} \geq \lambda_{2} \geq \ldots \geq \lambda_{\ell}\right) \in \mathbb{Y}_{\frac{\ell(N-\ell)}{2}}^{\ell}
$$

we have

$$
\begin{aligned}
& \lambda_{1}^{\top}=\ldots=\lambda_{\lambda_{\ell}}^{\top}=\ell \\
& \lambda_{\lambda_{\ell}+1}^{\top}=\ldots=\lambda_{\lambda_{\ell-1}}^{\top}=\ell-1 \\
& \lambda_{\lambda_{\ell-1}+1}^{\top}=\ldots=\lambda_{\lambda_{\ell-2}}^{\top}=\ell-2
\end{aligned}
$$

and so, since

$$
\begin{aligned}
k_{i}=N-1-k_{\ell+1-i} & =N-1-\left(\lambda_{\ell+1-i}+\ell-(\ell+1-i)\right) \\
& =N-\left(\lambda_{\ell+1-i}+i\right)
\end{aligned}
$$

we have, on the one hand

$$
k_{1}=N-\lambda_{\ell}-1>k_{2}=N-\lambda_{\ell-1}-2>k_{3}=N-\lambda_{\ell-2}-3,
$$

and on the other hand, using (6.0.4) and (6.0.8),

$$
\begin{aligned}
& k_{1}^{\top}=N-1>k_{2}^{\top}=N-2>\ldots>k_{\alpha}^{\top}=N-\alpha>\ldots>k_{\lambda_{\ell}}^{\top}=N-\lambda_{\ell}> \\
& k_{\lambda_{\ell}+1}^{\top}=N-\lambda_{\ell}-2>\ldots>k_{\beta}^{\top}=N-\beta-1>\ldots>k_{\lambda_{\ell-1}}^{\top}=N-\lambda_{\ell-1}-1> \\
& k_{\lambda_{\ell-1}+1}^{\top}=N-\lambda_{\ell-1}-3>\ldots>k_{\gamma}^{\top}=N-\gamma-2>\ldots>k_{\lambda_{\ell-2}}^{\top}=N-\lambda_{\ell-2}-2>\ldots
\end{aligned}
$$

So the gaps in $(6.0 .10$ coincide with the sequence (6.0.9). This ends the proof of Lemma 6.2. 
Proof of Proposition 6.1: One checks, using Proposition 3.2,

$$
\begin{aligned}
& \tilde{\tau}_{\ell}(t)=\sum_{\lambda \in \mathbb{Y}_{\frac{\ell(N-\ell)}{2}}^{(\ell)}} \prod_{1}^{\frac{\ell-1}{2}} b_{\lambda_{i}-i+\ell-\frac{N+1}{2}} \mathbf{s}_{\lambda}(t) \\
& =(-1)^{|\lambda|} \sum_{\lambda \in \mathbb{Y}_{\frac{\ell(N-\ell)}{2}}^{(\ell)}} \prod_{1}^{\frac{\ell-1}{2}} b_{k_{i}-\frac{N+1}{2}} \mathbf{s}_{\lambda^{\top}}(-t) \\
& =(-1)^{|\lambda|} \prod_{0}^{\frac{N-3}{2}} b_{i} \sum_{\lambda \in \mathbb{Y}_{\frac{\ell(N-\ell)}{2}}^{(\ell)}} \frac{1}{\prod_{1}^{\frac{N-\ell}{2}} b_{k_{i}^{\top}-\frac{N+1}{2}}} \mathbf{s}_{\lambda^{\top}}(-t), \text { using Lemma 6.2, } \\
& =(-1)^{|\lambda|} \prod_{0}^{\frac{N-3}{2}} b_{i} \sum_{\lambda^{\top} \in \mathbb{Y}_{\frac{((N-\ell)}{2}}^{(N-\ell)}} \prod_{1}^{\frac{N-\ell}{2}} b_{\lambda_{i}^{\top}-i+\ell-\frac{N+1}{2}}^{-1} \mathbf{s}_{\lambda^{\top}}(-t) \\
& =(-1)^{\frac{\ell(N-\ell)}{2}} \prod_{0}^{\frac{N-3}{2}} b_{i}\left(\left.\tau_{N-\ell}(-t)\right|_{b_{i} \rightarrow b_{i}^{-1}}\right) \text {, }
\end{aligned}
$$

which is, using Theorem 1.1, the $\tau$-function (modulo a constant) for the case where $N$ is odd and $N-\ell$ even, concluding the proof of the Proposition.

\section{Examples}

Example 1: Rectangular Jack polynomials

Proposition 7.1 When

$$
\begin{aligned}
b_{i} & =2 i+1 \text { for } N \text { even } \\
& =2 i+2 \text { for } N \text { odd }
\end{aligned}
$$


then the $\tau_{2 n}(t)$ 's are Jack polynomials for rectangular partitions, with $n \leq$ $[N / 2]$,

$$
\begin{aligned}
\tau_{2 n}(t) & =p f m_{2 n}(t) \\
& =\sum_{\lambda \in \mathbb{Y}_{n(N-2 n)}^{(2 n)}} \prod_{1}^{n}\left(k_{i}-k_{2 n+1-i}\right) \mathbf{s}_{\lambda}(t), \text { where } k_{i}=\lambda_{i}-i+2 n \\
& =\left.J_{\lambda}^{(1 / 2)}(x)\right|_{t_{i}=\frac{1}{i} \sum_{k} x_{k}^{i}} \text { for the partition } \lambda=(N-2 n)^{n} \\
& =\frac{1}{n !} \int_{\mathbb{R}^{n}} \Delta(z)^{4} \prod_{k=1}^{n} e^{2 \sum^{1} t_{i} z_{k}^{i}} \delta^{(N-2)}\left(z_{k}\right) d z_{k} .
\end{aligned}
$$

Then

$$
m_{\ell}(t)=E_{\ell, N}(t) m_{N}(0) E_{\ell, N}^{\top}(t)
$$

with

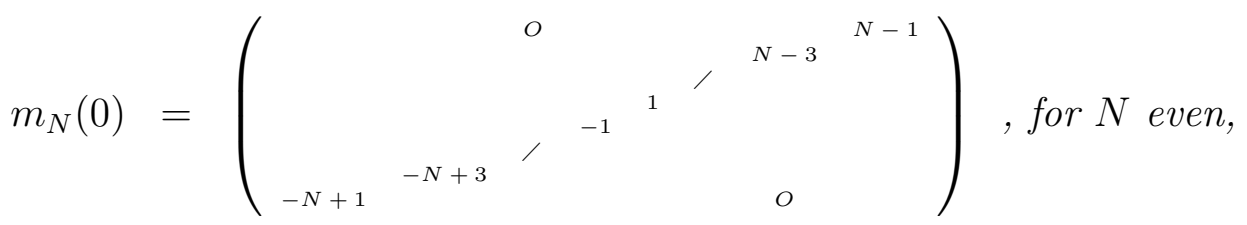

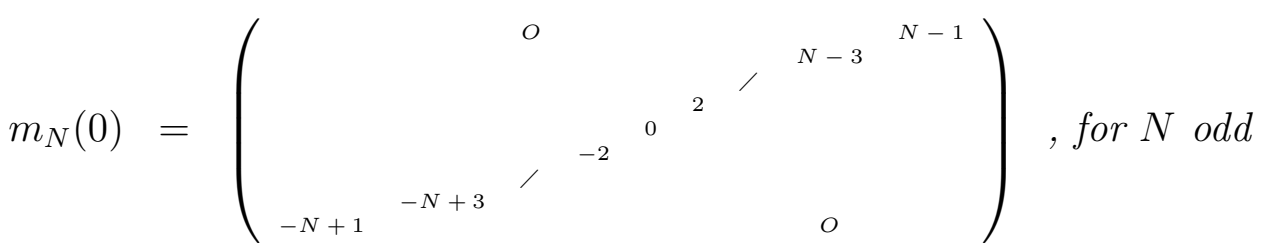


where (setting $\tilde{\mathbf{s}}_{n}(t)=\mathbf{s}_{n}(2 t)$ )

$m_{N}(t)$
$\quad=\left((j-i) \tilde{\mathbf{s}}_{N-i-j-1}\right)_{0 \leq i, j \leq N-1}$

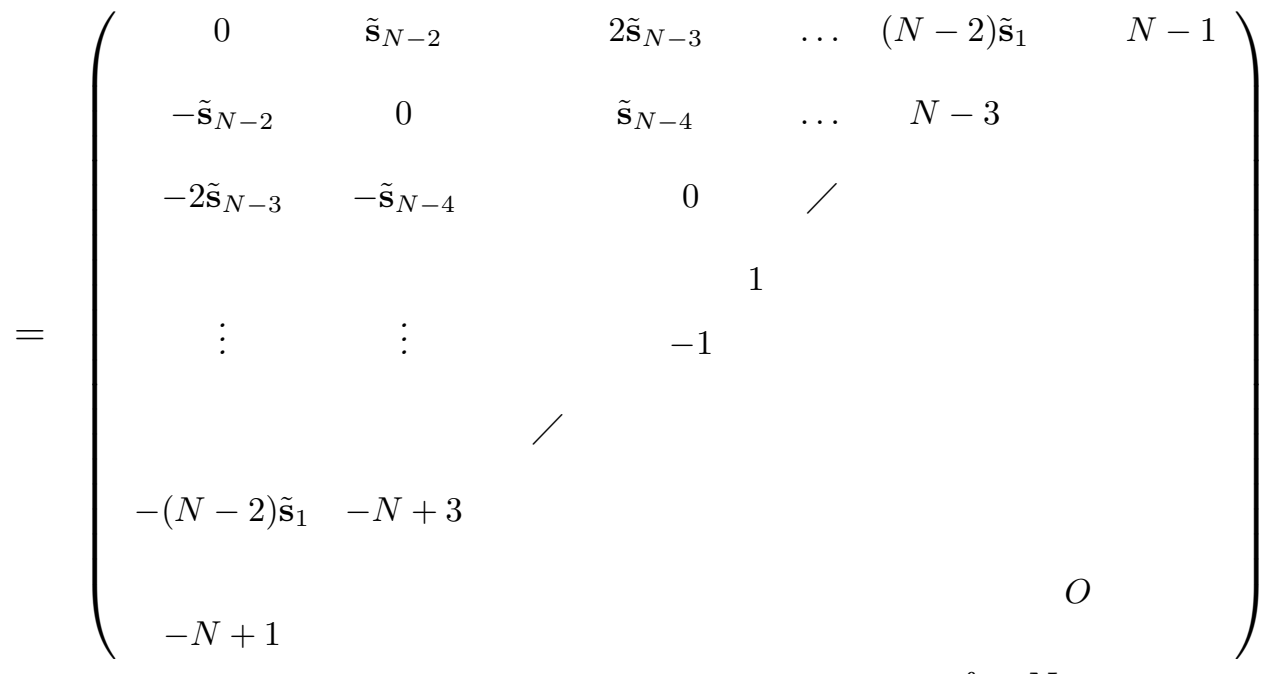

for $N$ even
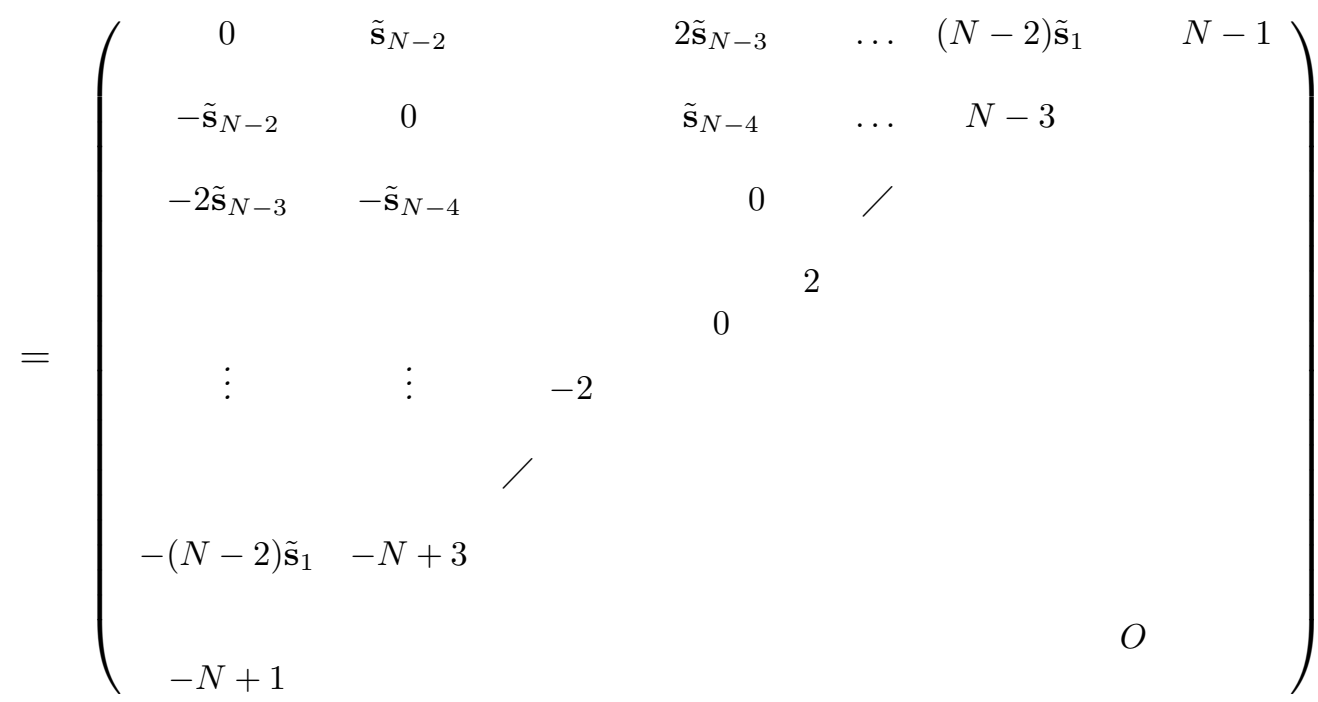

for $N$ odd 
Proof: Setting

$$
t_{k}=\frac{1}{k} \sum_{i=1}^{\ell} x_{i}^{k}
$$

we have

$$
\begin{aligned}
e^{\beta \sum_{1}^{\infty} t_{k} z^{k}} & =e^{\beta \sum_{i=1}^{\ell} \sum_{k=1}^{\infty} \frac{1}{k}\left(x_{i} z\right)^{k}} \\
& =\prod_{i=1}^{\ell}\left(\sum^{\sum_{k=1}^{\infty} \frac{1}{k}\left(x_{i} z\right)^{k}}\right)^{\beta} \\
& =\prod_{i=1}^{\ell}\left(1-x_{i} z\right)^{-\beta}
\end{aligned}
$$

According to Awata et al. [4], the Jack polynomials for rectangular partitions $s^{n}$ have the following integral representation: (for connections with random matrix theory, see [10])

$$
\begin{aligned}
c J_{s^{n}}^{1 / \beta} & =\oint_{z_{1}=\ldots=z_{n}=0}|\Delta(z)|^{2 \beta} \prod_{j=1}^{n} z_{j}^{-(n-1) \beta-s} \prod_{i=1}^{\ell}\left(1-x_{i} z_{j}\right)^{-\beta} \frac{d z_{j}}{2 \pi i z_{j}} \\
& =\oint_{z_{1}=\ldots=z_{n}=0}|\Delta(z)|^{2 \beta} \prod_{j=1}^{n} z_{j}^{-(n-1) \beta-s} e^{\beta \sum_{k=1}^{\infty} t_{k} z_{j}^{k}} \frac{d z_{j}}{2 \pi i z_{j}} \\
& =c_{n} \int_{\mathbb{R}^{n}}\left|\Delta_{n}(z)\right|^{2 \beta} \prod_{j=1}^{n} e^{\beta \sum_{k=1}^{\infty} t_{k} z_{j}^{k}} \delta^{s+(n-1) \beta}\left(z_{j}\right) d z_{j} .
\end{aligned}
$$


Setting $\beta=2, s=N-2 n$ and $2 \leq 2 n \leq N$ in the last integral, we have, using the standard derivation of the "symplectic" matrix integral, (see [2])

$$
\begin{aligned}
& \frac{1}{n !} \int_{\mathbb{R}^{n}} \Delta_{n}^{4}(z) \prod_{k=1}^{n} e^{2 \sum_{k=1}^{\infty} t_{k} z_{j}^{k}} \delta^{N-2}\left(z_{j}\right) d z_{j} \\
& \quad=p f\left(\int_{\mathbb{R}}\left\{y^{k}, y^{\ell}\right\} e^{2 \sum_{i=1}^{\infty} t_{i} y^{i}} \delta^{(N-2)}(y) d y\right)_{0 \leq k, \ell \leq 2 n-1} \\
& \quad=p f\left((k-\ell) \int_{\mathbb{R}} y^{k+\ell-1} e^{\left.2 \sum_{i=1}^{\infty} t_{i} y^{i} \delta^{(N-2)}(y) d y\right)_{0 \leq k, \ell \leq 2 n-1}}\right. \\
& \quad=p f\left((k-\ell) \sum_{i=0}^{\infty} \tilde{\mathbf{s}}_{i}(t) \int_{\mathbb{R}} y^{i+k+\ell-1} \delta^{(N-2)}(y) d y\right) \\
& \quad=p f\left((-1)^{N-2}(N-2) !(k-\ell) \tilde{\mathbf{s}}_{N-1-k-\ell}(t)\right)_{0 \leq k, \ell \leq 2 n-1} \\
& \quad=c_{N, n} p f\left((\ell-k) \tilde{\mathbf{s}}_{N-1-k-\ell}(t)\right)_{0 \leq k, \ell \leq 2 n-1}
\end{aligned}
$$

In order to find the initial condition $m_{N}(0)$, one sets $t=0$ in the last matrix appearing in (7.0.3), to yield

$$
\left((\ell-k) \tilde{\mathbf{s}}_{N-1-k-\ell}(0)\right)_{0 \leq k, \ell \leq N-1} .
$$

All entries of this matrix vanish, except the antidiagonal, from which one reads off the $b_{i}$ 's:

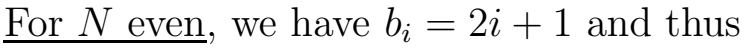

$$
\begin{aligned}
b_{\lambda_{i}-i+\ell-N / 2} & =2\left(\lambda_{i}-i+\ell-\frac{N}{2}\right)+1 \\
& =\lambda_{i}-\lambda_{\ell+1-i}-2 i+\ell+1 \quad \text { using } \lambda_{i}+\lambda_{\ell+1-i}=N-\ell \\
& =k_{i}-k_{\ell+1-i} \quad \text { using } k_{i}=\lambda_{i}-i+2 n .
\end{aligned}
$$

For $N$ odd, we have $b_{i}=2 i+2$ and thus

$$
\begin{aligned}
b_{\lambda_{i}-i+\ell-(N+1) / 2} & =2\left(\lambda_{i}-i+\ell-\frac{N+1}{2}\right)+2 \\
& =\lambda_{i}-\lambda_{\ell+1-i}-2 i+\ell+1 \quad \text { using } \lambda_{i}+\lambda_{\ell+1-i}=N-\ell \\
& =k_{i}-k_{\ell+1-i},
\end{aligned}
$$

ending the proof of Proposition 7.1. 
Example: For $n=4$ and $b_{0}=1, b_{1}=3$, the solution to the system (1.0.8) is given by

$$
L=\frac{1}{\left(t_{2}+t_{1}^{2}\right)^{2}}\left(\begin{array}{cccc}
0 & 1 & 0 & 0 \\
t_{1} & 2\left(t_{2}-t_{1}^{2}\right) & -\sqrt{3} t_{1} & 0 \\
\frac{2}{\sqrt{3}}\left(t_{2}-t_{1}^{2}\right) & -\frac{16}{\sqrt{3}} t_{1} t_{2} & -2\left(t_{2}-t_{1}^{2}\right) & 1 \\
-\sqrt{3} t_{1} & -2 \sqrt{3}\left(t_{2}-t_{1}^{2}\right) & 3 t_{1} & 0
\end{array}\right)
$$

Indeed

$$
m_{4}=\left(\begin{array}{cccc}
0 & -\tilde{\mathbf{s}}_{2} & -2 \tilde{\mathbf{s}}_{1} & -3 \\
\tilde{\mathbf{s}}_{2} & 0 & -1 & 0 \\
2 \tilde{\mathbf{s}}_{1} & 1 & 0 & 0 \\
3 & 0 & 0 & 0
\end{array}\right)=Q^{-1} J Q^{\top-1}
$$

with

$$
Q=D\left(\begin{array}{cccc}
1 & 0 & 0 & 0 \\
0 & 1 & 0 & 0 \\
1 & -2 \tilde{\mathbf{s}}_{1} & \tilde{\mathbf{s}}_{2} & 0 \\
0 & -3 & 0 & \tilde{\mathbf{s}}_{2}
\end{array}\right)
$$

where

$$
D=\operatorname{diag}\left(\frac{1}{\sqrt{\widetilde{\mathbf{s}}_{2}}}, \frac{1}{\sqrt{\widetilde{\mathbf{s}}_{2}}}, \frac{1}{\sqrt{3 \tilde{\mathbf{s}}_{2}}}, \frac{1}{\sqrt{3 \tilde{\mathbf{s}}_{2}}}\right)
$$

Therefore

$$
\begin{aligned}
L & =Q \Lambda Q^{-1} \\
& =\frac{1}{\tilde{\mathbf{s}}_{2}^{2}}\left(\begin{array}{cccc}
0 & 1 & 0 & 0 \\
2 \tilde{\mathbf{s}}_{1} & 4\left(\tilde{\mathbf{s}}_{2}-\tilde{\mathbf{s}}_{1}^{2}\right) & -2 \sqrt{3} \tilde{\mathbf{s}}_{1} & 0 \\
\frac{4}{\sqrt{3}}\left(\tilde{\mathbf{s}}_{2}-\tilde{\mathbf{s}}_{1}^{2}\right) & -\frac{8 \tilde{\mathbf{s}}_{1}}{\sqrt{3}}\left(2 \tilde{\mathbf{s}}_{2}-\tilde{\mathbf{s}}_{1}^{2}\right) & -4\left(\tilde{\mathbf{s}}_{2}-\tilde{\mathbf{s}}_{1}^{2}\right) & 1 \\
-\frac{6}{\sqrt{3}} \tilde{\mathbf{s}}_{1} & -\frac{12}{\sqrt{3}}\left(\tilde{\mathbf{s}}_{2}-\tilde{\mathbf{s}}_{1}^{2}\right) & 6 \tilde{\mathbf{s}}_{1} & 0
\end{array}\right),
\end{aligned}
$$

leads to formula (7.0.5). 


\section{Example 2: Two-column Jack polynomials}

Proposition 7.2 For even N, choosing

$$
\left\{\begin{array}{l}
b_{0}=\ldots=b_{\frac{p}{2}-1}=0 \\
b_{\frac{p}{2}+k}=\frac{(1-\alpha)_{k}(p+1)_{k}}{k !(\alpha+p+1)_{k}}, \text { for } k=0, \ldots, \frac{N-2-p}{2},
\end{array}\right.
$$

one finds the most general two-row Jack polynomial for $\tau_{2}$, for arbitrary $\alpha$,

$$
\begin{aligned}
\tau_{2}(t) & =p f m_{2}(t) \\
& =J_{\left(\frac{N+p-2}{2}, \frac{N-p-2}{2}\right)}^{(1 / \alpha)}(t / \alpha) \\
& =c \oint \frac{d x}{2 \pi i} \frac{d y}{2 \pi i} \frac{(y-x)^{2 \alpha}}{(x y)^{\alpha+\frac{N}{2}}} e^{\sum_{1}^{\infty} t_{i}\left(x^{i}+y^{i}\right)}\left(\frac{x}{y}\right)^{p / 2}{ }_{2} F_{1}\left(\alpha,-p ; 1-\alpha-p ; \frac{y}{x}\right)
\end{aligned}
$$

and for general $\ell \geq 2$,

$$
\begin{aligned}
& \tau_{\ell}(t)=\frac{2 c}{\ell ! !} \oint \frac{\left(z_{2}-z_{1}\right)^{2 \alpha-1}}{z_{2}\left(z_{1} z_{2}\right)^{\alpha-1}}\left(\frac{z_{1}}{z_{2}}\right)^{p / 2}{ }_{2} F_{1}\left(\alpha,-p ; 1-\alpha-p ; \frac{z_{2}}{z_{1}}\right) \\
& \frac{\prod_{i=2}^{\ell / 2} \rho\left(z_{2 i} / z_{2 i-1}\right)}{\prod_{i=1}^{\ell / 2} z_{2 i-1}^{\frac{N}{2}-2 i+3} z_{2 i}^{\frac{N}{2}-2 i+1}} \prod_{1 \leq i<j \leq \ell}\left(1-\frac{z_{i}}{z_{j}}\right) \prod_{j=1}^{\ell} e^{\sum_{k=1}^{\infty} t_{k} z_{j}^{k}} \frac{d z_{j}}{2 \pi i}
\end{aligned}
$$

where

$$
\rho(x)=\sum_{i=0}^{\frac{N-2}{2}} b_{i}\left(x^{-i-1}-x^{i}\right)
$$

$$
{ }^{13}(a)_{k}=\frac{\Gamma(a+k)}{\Gamma(a)}=a(a+1) \ldots(a+k-1)
$$


Proof: According to a formula by Stanley [9], two-column Jack polynomials can be expressed as a linear combination of two-column Schur polynomials. So, setting in the end $2 s=N-2-p$, we have

$$
\begin{aligned}
\tau_{2}(t) & =\sum_{k=0}^{\frac{N-2}{2}} b_{k} \mathbf{s}_{\frac{N-2}{2}+k, \frac{N-2}{2}-k}(t), \text { with } b_{k} \text { as in }(7.0 .6) \\
& =\sum_{k=\frac{p}{2}}^{\frac{N-2}{2}} \frac{(1-\alpha)_{k-p / 2}(p+1)_{k-p / 2}(-1)^{N-2}}{(k-p / 2) !(\alpha+p+1)_{k-p / 2}} \mathbf{s}_{\frac{N-2}{2}+k, \frac{N-2}{2}-k}(t) \\
& =\sum_{k=\frac{p}{2}}^{\frac{N-2}{2}} \frac{(1-\alpha)_{k-p / 2}(p+1)_{k-p / 2}}{(k-p / 2) !(\alpha+p+1)_{k-p / 2} \mathbf{s}^{\frac{N-2}{2}-k} 1^{2 k}}(-t) \\
& =\sum_{k=0}^{\frac{N-p-2}{2}} \frac{(1-\alpha)_{k}(p+1)_{k}}{k !(\alpha+p+1)_{k}} \mathbf{s}_{2^{\frac{N-2}{2}-k-p / 2} 1^{2 k+p}}(-t) \\
& =\sum_{k=0}^{s} \frac{(1-\alpha)_{k}(p+1)_{k}}{k !(\alpha+p+1)_{k}} \mathbf{s}_{2^{s-k} 1^{2 k+p}}(-t) \\
& =J_{2^{s} 1^{p}}^{(\alpha)}(-t) \quad(\text { Stanley's formula }) \\
& =J_{(p+s, s)}^{(1 / \alpha)}(t / \alpha) \quad \text { using duality, }
\end{aligned}
$$

showing that any two-row Jack polynomial can serve as Pfaff $\tau$-function $\tau_{2}$.

According to [⿴囗十], Jack polynomials also have an integral representation, and so $\tau_{2}(t)$ can also be expressed as

$$
\begin{aligned}
\tau_{2}(t) & =J_{(p+s, s)}^{(1 / \alpha)}(t / \alpha) \\
& =c^{\prime} \oint \frac{d x}{2 \pi i x} \frac{d y}{2 \pi i y} \frac{d z}{2 \pi i z} \frac{(x-y)^{2 \alpha}(x y)^{-s} z^{-p}}{((x-z)(y-z))^{\alpha}} e^{\sum_{1}^{\infty} t_{i}\left(x^{i}+y^{i}\right)} \\
& =\left.c^{\prime} \oint \frac{d x}{2 \pi i x} \frac{d y}{2 \pi i y}(x-y)^{2 \alpha}(x y)^{-s} e^{\sum_{1}^{\infty} t_{i}\left(x^{i}+y^{i}\right)} D_{z}^{p}((x-z)(y-z))^{-\alpha}\right|_{z=0} \\
& =c^{\prime}(\alpha)_{p} \oint \frac{d x}{2 \pi i x} \frac{d y}{2 \pi i y} \frac{(x-y)^{2 \alpha}}{(x y)^{\alpha+s} y^{p}} e^{\sum_{1}^{\infty} t_{i}\left(x^{i}+y^{i}\right)}{ }_{2} F_{1}\left(\alpha,-p ; 1-\alpha-p ; \frac{y}{x}\right),
\end{aligned}
$$


where we used the identity:

$$
\begin{aligned}
& \left.D_{z}^{p}((x-z)(y-z))^{-\alpha}\right|_{z=0} \\
& =\left.(x y)^{-\alpha} D_{z}^{p}\left(\left(1-\frac{z}{x}\right)^{-\alpha}\left(1-\frac{z}{y}\right)^{-\alpha}\right)\right|_{z=0} \\
& =\left.(x y)^{-\alpha} D_{z}^{p}\left(\sum_{k, \ell=0}^{\infty} \frac{(\alpha)_{k}(\alpha)_{\ell} z^{k+\ell}}{k ! \ell !} x^{k} y^{\ell}\right)\right|_{z=0} \\
& =p !(x y)^{-\alpha} \sum_{k+\ell=p} \frac{(\alpha)_{k}(\alpha)_{\ell}}{k ! \ell !} x^{-k} y^{-\ell} \\
& \left.=p !(x y)^{-\alpha} y^{-p} \sum_{k=0}^{p} \frac{(\alpha)_{k}(\alpha)_{p-k}}{k !(p-k) !} \frac{y}{x}\right)^{k} \\
& =(\alpha)_{p}(x y)^{-\alpha} y^{-p} \sum_{k=0}^{p} \frac{(\alpha)_{k}(-p)_{k}}{k !(1-\alpha-p)_{k}}\left(\frac{y}{x}\right)^{k} \\
& =(\alpha)_{p}(x y)^{-\alpha} y^{-p}{ }_{2} F_{1}\left(\alpha,-p ; 1-\alpha-p ; \frac{y}{x}\right) .
\end{aligned}
$$

This proves identity (7.0.7).

Applying Theorem 1.3, we find the higher $\tau_{\ell}$ 's, by applying the integrated vertex operator

$$
Y_{\frac{N-2}{2}-2 j}(t)=\frac{1}{(2 \pi i)^{2}} \oint_{\infty} \oint_{\infty} X\left(t ; z_{2 j+2}\right) X\left(t ; z_{2 j+1}\right) \frac{\rho_{b}\left(z_{2 j+2} / z_{2 j+1}\right) d z_{2 j+2} d z_{2 j+1}}{z_{2 j+1}^{2}\left(z_{2 j+1} z_{2 j+2}\right)^{\frac{N-2}{2}-2 j}}
$$


for $j=1,2, \ldots,(\ell-2) / 2$ to $\tau_{2}$ (see formula $\left.(7.0 .7)\right)$; so, one finds

$$
\begin{aligned}
\tau_{\ell}= & \frac{2}{\ell ! !} Y_{\frac{N}{2}-\ell+1} \ldots Y_{\frac{N}{2}-5} Y_{\frac{N}{2}-3} \tau_{2} \\
= & \frac{2 c^{\prime}(\alpha)_{p}}{\ell ! !} \oint \frac{\left(z_{2}-z_{1}\right)^{2 \alpha}}{\left(z_{1} z_{2}\right)^{\alpha+\frac{N}{2}}}\left(\frac{z_{1}}{z_{2}}\right)^{p / 2}{ }_{2} F_{1}\left(\alpha,-p ; 1-\alpha-p ; \frac{z_{2}}{z_{1}}\right) \\
& \frac{\rho\left(z_{\ell} / z_{\ell-1}\right) \ldots \rho\left(z_{4} / z_{3}\right)}{\left(z_{3} z_{5} \ldots z_{\ell-1}\right)^{2}\left(z_{3} z_{4}\right)^{\frac{N}{2}-3}\left(z_{5} z_{6}\right)^{\frac{N}{2}-5} \ldots\left(z_{\ell-1} z_{\ell}\right)^{\frac{N}{2}-\ell+1}} \\
& X\left(t ; z_{\ell}\right) X\left(t ; z_{\ell-1}\right) \ldots X\left(t ; z_{4}\right) X\left(t ; z_{3}\right) e^{\sum_{1}^{\infty} t_{k}\left(z_{1}^{k}+z_{2}^{k}\right)} \prod_{j=1}^{\ell} \frac{d z_{j}}{2 \pi i} \\
= & \frac{2 c^{\prime}(\alpha)_{p}}{\ell ! !} \oint \frac{\left(z_{2}-z_{1}\right)^{2 \alpha} z_{1}^{2}\left(z_{1} z_{2}\right)^{N / 2-1}}{\left(z_{1} z_{2}\right)^{\alpha+\frac{N}{2}}}\left(\frac{z_{1}}{z_{2}}\right)^{p / 2}{ }_{2} F_{1}\left(\alpha,-p ; 1-\alpha-p ; \frac{z_{2}}{z_{1}}\right) \\
& \left(1-\frac{z_{1}}{z_{2}}\right)^{-1} \frac{\rho\left(z_{\ell} / z_{\ell-1}\right) \ldots \rho\left(z_{4} / z_{3}\right)}{\ell / 2} \prod_{1 \leq i<j \leq \ell}\left(1-\frac{z_{i}}{z_{j}}\right) \prod_{j=1}^{\ell} e^{\sum_{1}^{\infty} t_{k} z_{j}^{k}} \frac{d z_{j}}{2 \pi i} \\
& \frac{\prod_{1}^{2} z_{2 i-1}^{2}\left(z_{2 i-1} z_{2 i}\right)^{\frac{N}{2}-2 i+1}}{\ell / 2} \prod_{1 \leq 2}\left(1-\frac{z_{i}}{z_{j}}\right) \prod_{j=1}^{\ell} e^{\sum_{k=1}^{\infty} t_{k} z_{j}^{k}} \frac{d z_{j}}{2 \pi i}, \\
& \frac{2 c^{\prime}(\alpha)_{p}}{\ell ! !} \oint \frac{\left(z_{2}-z_{1}\right)^{2 \alpha-1}}{z_{2}\left(z_{1} z_{2}\right)^{\alpha-1}}\left(\frac{z_{1}}{z_{2}}\right)^{p / 2}{ }_{2} F_{1}\left(\alpha,-p ; 1-\alpha-p ; \frac{z_{2}}{z_{1}}\right) \\
& \prod_{2 i}^{\frac{N}{2}-2 i+1}{ }_{1 \leq i<j \leq \ell}\left(z_{2 i} / z_{2 i-1}\right)
\end{aligned}
$$

establishing formula (7.0.8).

Alternative formula: The following formula has the advantage to be more

\footnotetext{
${ }^{14}$ replacing $x, y$ in $\tau_{2}$ with $z_{1}, z_{2}$.
} 
symmetric, but the disadvantage to have many more integrations:

$$
\tau_{\ell}(t)=\oint \prod_{i=1}^{\ell} \prod_{j=1}^{i} \frac{d z_{j}^{(i)}}{z_{j}^{(i)}} \prod_{i=1}^{\ell} e^{\sum_{1}^{\infty} t_{k}\left(z_{i}^{(\ell)}\right)^{-k}} \frac{\prod_{\substack { k=1 \\
\begin{subarray}{c}{1 \leq i, j \leq k \\
i \neq j{ k = 1 \\
\begin{subarray} { c } { 1 \leq i , j \leq k \\
i \neq j } }\end{subarray}} \prod_{\substack{\ell-1 \\
k=1}}\left(1-\frac{z_{i}^{(k)}}{z_{j}^{(k)}}\right)}{\substack{\begin{subarray}{c}{1 \leq i \leq k+1 \\
1 \leq j \leq k} }} \\
{1 \leq k}} K_{N, p, \ell}(Z)
$$

with

$$
\begin{aligned}
K_{N, p, \ell}= & \frac{\left(\prod_{j=1}^{\ell} z_{j}^{(\ell)}\right)^{\frac{N-p}{2}-1}\left(\prod_{j=1}^{\ell / 2} z_{j}^{(\ell / 2)}\right)^{p+1}}{\prod_{i=1}^{\ell-1} \prod_{1}^{i} z_{j}^{(i)}} \\
& \prod_{i=1}^{\ell / 2}{ }_{2} F_{1}\left(1-\alpha, p+1 ; 1+\alpha+p ; \frac{\prod_{j=1}^{i} z_{j}^{(i)} \prod_{j=1}^{\ell-i} z_{j}^{(\ell-i)}}{\prod_{j=1}^{\ell-1} z_{j}^{(i-1)} \prod_{j=1}^{\ell+i} z_{j}^{(\ell+1-i)}}\right)
\end{aligned}
$$

\section{References}

[1] M. Adler, T, Shiota and P. van Moerbeke: Pfaff $\tau$-functions, Math. Annalen, 322, 423-476 (2002) (arXiv: solv-int/9909010)

[2] M. Adler and P. van Moerbeke: Toda versus Pfaff Lattice and related polynomials, Duke Math. J., 112, 2002 (arXiv: solv-int/9912008)

[3] M. Adler, E. Horozov and P. van Moerbeke: The Pfaff lattice and skeworthogonal polynomials, Intern. Math. Res. Notices, 11, 569-588 (1999)

[4] H. Awata, Y. Matsuo, S. Odake and J. Shiraishi: Excited states of the Calogero-Sutherland model and singular vectors of the $W_{N}$ algebra, Nuclear Phys. B 449, 347-374 (1995)

[5] L. Dickey: Soliton equations and integrable systems, World Scientific (1991). 
[6] V.B. Kuznetsov and E.K. Sklyanin: Separation of variables in $A_{2}$ type Jack polynomials, RIMS Kokyuroku, 919, 27-34 (1995)

[7] V.B. Kuznetsov and E.K. Sklyanin: Factorisation of Macdonald polynomials, in: Symmetries and Integrability of Difference Equations, London Mathematical Society, Lecture Note Series, 255, 370-384 (1999); (Eds P.A. Clarkson and F.W. Nijhoff, Cambridge University Press).

[8] I.G. MacDonald: "Symmetric functions and Hall polynomials", Clarendon Press, 1995.

[9] R. P. Stanley: Some combinatorial properties of Jack symmetric functions, Adv. in Math., 77, 76-115 (1989).

[10] P. van Moerbeke: Integrable lattices: random matrices and random permutations, "Random matrices and their applications", Mathematical Sciences research Institute Publications \#40, Cambridge University Press, pp 321-406, (2001). 